\title{
Convergence of Pascal-Like Triangles in Parry-Bertrand Numeration Systems
}

\author{
Manon Stipulanti ${ }^{1}$ \\ University of Liège \\ Department of Mathematics \\ Allée de la Découverte 12 (B37) \\ 4000 Liège, Belgium \\ M.Stipulanti@uliege.be
}

\begin{abstract}
We pursue the investigation of generalizations of the Pascal triangle based on binomial coefficients of finite words. These coefficients count the number of times a finite word appears as a subsequence of another finite word. The finite words occurring in this paper belong to the language of a Parry numeration system satisfying the Bertrand property, i.e., we can add or remove trailing zeroes to valid representations. It is a folklore fact that the Sierpiński gasket is the limit set, for the Hausdorff distance, of a convergent sequence of normalized compact blocks extracted from the classical Pascal triangle modulo 2. In a similar way, we describe and study the subset of $[0,1] \times[0,1]$ associated with the latter generalization of the Pascal triangle modulo a prime number.
\end{abstract}

2010 Mathematics Subject Classification: 11A63, 11A67, 11B65, 11K16, 68R15.

Keywords: Binomial coefficients of words; generalized Pascal triangles; $\beta$-expansions; Perron numbers; Parry numbers; Bertrand numeration systems.

\section{Introduction}

Several generalizations and variations of the Pascal triangle exist and lead to interesting combinatorial, geometrical or dynamical properties [1, 2, 8, 9, 10. This paper is inspired by a series of papers based on generalizations of Pascal triangles to finite words [10, 11, 12, 13].

\subsection{Binomial coefficients of words and Pascal-like triangles}

In this short subsection, we briefly introduce the concepts we use in this paper. For more definitions, see section 2. A finite word is a finite sequence of letters belonging to a finite set called the alphabet. The binomial coefficient $\left(\begin{array}{l}u \\ v\end{array}\right)$ of two finite words $u$ and $v$ is the number of times $v$ occurs as a subsequence of $u$ (meaning as a "scattered" subword).

Let $A$ be a totally ordered alphabet, and let $L \subset A^{*}$ be an infinite language over $A$. We order the words of $L$ by increasing genealogical order and we write $L=\left\{w_{0}<w_{1}<w_{2}<\cdots\right\}$. Associated with the language $L$, we define a Pascal-like triangle $\mathrm{P}_{L}: \mathbb{N} \times \mathbb{N} \rightarrow \mathbb{N}$ represented as an infinite table. The entry $\mathrm{P}_{L}(m, n)$ on the $m$ th row and $n$th column of $\mathrm{P}_{L}$ is the integer $\left(\begin{array}{c}w_{m} \\ w_{n}\end{array}\right)$.

\footnotetext{
${ }^{1}$ Corresponding author.
} 


\subsection{Previous work}

Let $b$ be an integer greater than 1 . We let $\operatorname{rep}_{b}(n)$ denote the (greedy) base- $b$ expansion of $n \in \mathbb{N} \backslash\{0\}$ starting with a non-zero digit. We set $\operatorname{rep}_{b}(0)$ to be the empty word denoted by $\varepsilon$. We let

$$
L_{b}=\{1, \ldots, b-1\}\{0, \ldots, b-1\}^{*} \cup\{\varepsilon\}
$$

be the set of base- $b$ expansions of the non-negative integers. In [10], we study the particular case of $L=L_{b}$. The increasing genealogical order thus coincides with the classical order in $\mathbb{N}$. For example, see Table 1 for the first few values ${ }^{2}$ of $\mathrm{P}_{2}$. Clearly, $\mathrm{P}_{b}$ contains several subtables corresponding to the usual Pascal triangle.

\begin{tabular}{r|cccccccc} 
& $\varepsilon$ & 1 & 10 & 11 & 100 & 101 & 110 & 111 \\
\hline$\varepsilon$ & $\mathbf{1}$ & 0 & 0 & 0 & 0 & 0 & 0 & 0 \\
1 & $\mathbf{1}$ & $\mathbf{1}$ & 0 & 0 & 0 & 0 & 0 & 0 \\
10 & 1 & 1 & 1 & 0 & 0 & 0 & 0 & 0 \\
11 & $\mathbf{1}$ & $\mathbf{2}$ & 0 & $\mathbf{1}$ & 0 & 0 & 0 & 0 \\
100 & 1 & 1 & 2 & 0 & 1 & 0 & 0 & 0 \\
101 & 1 & 2 & 1 & 1 & 0 & 1 & 0 & 0 \\
110 & 1 & 2 & 2 & 1 & 0 & 0 & 1 & 0 \\
111 & $\mathbf{1}$ & $\mathbf{3}$ & 0 & $\mathbf{3}$ & 0 & 0 & 0 & $\mathbf{1}$
\end{tabular}

Table 1: The first few values in the generalized Pascal triangle $\mathrm{P}_{2}(\underline{\mathrm{A} 282714})$.

For instance, it contains $(b-1)$ copies of the usual Pascal triangle obtained when only considering words of the form $a^{m}$ with $a \in\{1, \ldots, b-1\}$ and $m \geq 0$ since $\left(\begin{array}{c}a^{m} \\ a^{n}\end{array}\right)=\left(\begin{array}{c}m \\ n\end{array}\right)$. In Table 1, a copy of the classical Pascal triangle is written in bold.

Considering the intersection of the lattice $\mathbb{N}^{2}$ with $\left[0,2^{n}\right] \times\left[0,2^{n}\right]$, the first $2^{n}$ rows and columns of the usual Pascal triangle modulo $2\left(\left(\begin{array}{c}i \\ j\end{array}\right) \bmod 2\right)_{0 \leq i, j<2^{n}}$ provide a coloring of this lattice. If we normalize this compact set by a homothety of ratio $1 / 2^{n}$, we get a sequence of subsets of $[0,1] \times[0,1]$ which converges, for the Hausdorff distance, to the Sierpiński gasket when $n$ tends to infinity. In the extended context described above, the case when $b=2$ gives similar results and the limit set, generalizing the Sierpiński gasket, is described using a simple combinatorial property called $(\star)$ [10].

Inspired by [10, we study the sequence $\left(S_{b}(n)\right)_{n \geq 0}$ which counts, on each row $m$ of $\mathrm{P}_{b}$, the number of words of $L_{b}$ occurring as subwords of the $m$ th word in $L_{b}$, i.e., $S_{b}(m)=\#\left\{n \in \mathbb{N} \mid \mathrm{P}_{b}(m, n)>0\right\}$. This sequence is shown to be $b$-regular [11, 13]. We also consider the summatory function $\left(A_{b}(n)\right)_{n \geq 0}$ of the sequence $\left(S_{b}(n)\right)_{n \geq 0}$ and study its behavior 12 , 13 .

So far, the setting is the one of integer bases. As a first extension, we handle the case of the Fibonacci numeration system, i.e., with the language $L_{F}=\{\varepsilon\} \cup 1\{0,01\}^{*}$ [11, 12]. It turns out that the sequence $\left(S_{F}(n)\right)_{n \geq 0}$ counting the number of words in $L_{F}$ occurring as subwords of the $n$th word in $L_{F}$ has properties similar to those of $\left(S_{b}(n)\right)_{n \geq 0}$. Finally, the summatory function $\left(A_{F}(n)\right)_{n \geq 0}$ of the sequence $\left(S_{F}(n)\right)_{n \geq 0}$ has a behavior similar to the one of $\left(A_{b}(n)\right)_{n \geq 0}$.

\subsection{Our contribution}

The Fibonacci numeration system belongs to an extensively studied family of numeration systems called Parry-Bertrand numeration systems, which are based on particular sequences $(U(n))_{n \geq 0}$ (the precise definitions are given later). In this paper, we fill the gap between integer bases and the Fibonacci numeration systems by extending the results of [10] to every Parry-Bertrand numeration system. First, we generalize

\footnotetext{
${ }^{2}$ Some of the objects discussed here are stored in Sloane's On-Line Encyclopedia of Integer Sequences [20]. See sequences A007306, A282714 A282715 A282720, A282728, A284441, and A284442
} 
the construction of Pascal-like triangles to every Parry-Bertrand numeration system. For a given ParryBertrand numeration system based on a particular sequence $(U(n))_{n \geq 0}$, we consider the intersection of the lattice $\mathbb{N}^{2}$ with $[0, U(n)] \times[0, U(n)]$. Then the first $U(n)$ rows and columns of the corresponding generalized Pascal triangle modulo 2 provide a coloring of this lattice regarding the parity of the corresponding binomial coefficients. If we normalize this compact set by a homothety of ratio $1 / U(n)$, we get a sequence in $[0,1] \times[0,1]$ which converges, for the Hausdorff distance, to a limit set when $n$ tends to infinity. Again, the limit set is described using a simple combinatorial property extending the one from 10 .

Compared to the integer bases, new technicalities have to be taken into account to generalize Pascal triangles to a large class of numeration systems. The numeration systems occurring in this paper essentially have two properties. The first one is that the language of the numeration system comes from a particular automaton. The second one is the Bertrand condition which allows to delete or add ending zeroes to valid representations.

This paper is organized as follows. In Section 2 , we collect necessary background. Section 3 is devoted to a special combinatorial property that extends the $(\star)$ condition from [10]. This new condition allows us to define a sequence of compact sets, which is shown to be a Cauchy sequence in Section 4 . In Section 5 using the property of the latter sequence, we define a limit set which is the analogue of the Sierpiński gasket in the classical framework. We show that the sequence of subblocks of the generalized Pascal triangle modulo 2 in a Parry-Bertrand numeration converges to this new limit set. As a final remark, we consider the latter sequence of compact sets modulo any prime number.

\section{Background and particular framework}

We begin this section with well-known definitions from combinatorics on words; see, for instance, [18. Let $A$ be an alphabet, i.e., a finite set. The elements of $A$ are called letters. A finite sequence over $A$ is called a finite word. The length of a finite word $w$, denoted by $|w|$, is the number of letters belonging to $w$. The only word of length 0 is the empty word $\varepsilon$. The set of finite words over the alphabet $A$ including the empty word (resp., excluding the empty word) is denoted by $A^{*}$ (resp., $A^{+}$). The set of words of length $n$ over $A$ is denoted by $A^{n}$. If $u$ and $v$ are two finite words belonging to $A^{*}$, the binomial coefficient $\left(\begin{array}{l}u \\ v\end{array}\right)$ of $u$ and $v$ is the number of occurrences of $v$ as a subsequence of $u$, meaning as a scattered subword. The sequences over $A$ indexed by $\mathbb{N}$ are the infinite words over $A$. If $w$ is a finite non-empty word over $A$, we let $w^{\omega}:=w w w \cdots$ denote the infinite word obtained by concatenating infinitely many copies of $w$. If $L \subset A^{*}$ is a set of finite words and $u \in A^{*}$ is a finite word, we let $u^{-1}$. $L$ denote the set of words $\left\{v \in A^{*} \mid u v \in L\right\}$. Let $A$ be totally ordered. If $u, v \in A^{*}$ are two words, we say that $u$ is less than $v$ in the genealogical order and we write $u<v$ if either $|u|<|v|$, or if $|u|=|v|$ and there exist words $p, q, r \in A^{*}$ and letters $a, b \in A$ with $u=p a q, v=p b r$ and $a<b$. By $u \leq v$, we mean that either $u<v$, or $u=v$.

In the first part of this section, we gather two results on binomial coefficients of finite words and integers. For a proof of the first lemma, we refer the reader to [14, Chap. 6].

Lemma 1. Let $A$ be a finite alphabet. Let $u, v \in A^{*}$ and let $a, b \in A$. Then we have

$$
\left(\begin{array}{c}
u a \\
v b
\end{array}\right)=\left(\begin{array}{c}
u \\
v b
\end{array}\right)+\delta_{a, b}\left(\begin{array}{l}
u \\
v
\end{array}\right)
$$

where $\delta_{a, b}$ is equal to 1 if $a=b, 0$ otherwise.

Let us also recall Lucas' theorem relating classical binomial coefficients modulo a prime number $p$ with base- $p$ expansions. See [16, p. 230] or [7. Note that in the following statement, if the base- $p$ expansions of $m$ and $n$ are not of the same length, then we pad the shortest with leading zeroes.

Theorem 2. Let $m$ and $n$ be two non-negative integers and let $p$ be a prime number. If

$$
m=m_{k} p^{k}+m_{k-1} p^{k-1}+\cdots+m_{1} p+m_{0}
$$


and

$$
n=n_{k} p^{k}+n_{k-1} p^{k-1}+\cdots+n_{1} p+n_{0}
$$

with $m_{i}, n_{i} \in\{0, \ldots, p-1\}$ for all $i$, then the following congruence relation holds

$$
\left(\begin{array}{c}
m \\
n
\end{array}\right) \equiv \prod_{i=0}^{k}\left(\begin{array}{c}
m_{i} \\
n_{i}
\end{array}\right) \bmod p
$$

using the following convention: $\left(\begin{array}{c}m \\ n\end{array}\right)=0$ if $m<n$.

In the last part of this section, we introduce the setting of particular numeration systems that are used in this paper: the Parry-Bertrand numeration systems. First of all, we recall several definitions and results about representations of real numbers. For more details, see, for instance, [3, Chap. 2], [15, Chap. 7] or [19.

Definition 3. Let $\beta \in \mathbb{R}_{>1}$ and let $A_{\beta}=\{0,1, \ldots,\lceil\beta\rceil-1\}$. Every real number $x \in[0,1)$ can be written as a series

$$
x=\sum_{j=1}^{+\infty} c_{j} \beta^{-j}
$$

where $c_{j} \in A_{\beta}$ for all $j \geq 1$, and where $\lceil\cdot\rceil$ denotes the ceiling function defined by $\lceil x\rceil=\inf \{z \in \mathbb{Z} \mid z \geq x\}$. The infinite word $c_{1} c_{2} \cdots$ is called a $\beta$-representation of $x$. Among all the $\beta$-representations of $x$, we define the $\beta$-expansion $d_{\beta}(x)$ of $x$ obtained in a greedy way, i.e., for all $j \geq 1$, we have $c_{j} \beta^{-j}+c_{j+1} \beta^{-j-1}+\cdots<\beta^{-j+1}$. We also make use of the following convention: if $w=w_{n} \cdots w_{0}$ is a finite word (resp., $w=w_{1} w_{2} \cdots$ is an infinite word) over $A_{\beta}$, the notation $0 . w$ has to be understood as the real number $\sum_{j=0}^{n} w_{j} \beta^{j-n-1}$ (resp., $\left.\sum_{j=1}^{+\infty} w_{j} \beta^{-j}\right)$; it actually corresponds to the value of the word $w$ in base $\beta$.

In an analogous way, the $\beta$-expansion $d_{\beta}(1)$ of 1 the following infinite word over $A_{\beta}$

$$
d_{\beta}(1):=\left\{\begin{array}{cl}
(\beta-1)^{\omega}, & \text { if } \beta \in \mathbb{N} \\
(\lceil\beta\rceil-1) d_{\beta}(1-(\lceil\beta\rceil-1) / \beta), & \text { otherwise. }
\end{array}\right.
$$

In other words, if $\beta$ is not an integer, the first digit of the $\beta$-expansion of 1 is $\lceil\beta\rceil-1$ and the other digits are derived from the $\beta$-expansion of $1-(\lceil\beta\rceil-1) / \beta$.

Let $d_{\beta}(1)=\left(t_{n}\right)_{n \geq 1}$ be the $\beta$-expansion of 1 . Observe that $t_{1}=\lceil\beta\rceil-1$. We define the quasi-greedy $\beta$-expansion $d_{\beta}^{*}(1)$ of 1 as follows. If $d_{\beta}(1)=t_{1} \cdots t_{m}$ is finite, i.e., $t_{m} \neq 0$ and $t_{j}=0$ for all $j>m$, then $d_{\beta}^{*}(1)=\left(t_{1} \cdots t_{m-1}\left(t_{m}-1\right)\right)^{\omega}$, otherwise $d_{\beta}^{*}(1)=d_{\beta}(1)$.

A real number $\beta>1$ is a Parry number if $d_{\beta}(1)$ is ultimately periodic. If $d_{\beta}(1)$ is finite, then $\beta$ is called a simple Parry number. In this case, Proposition 5 gives an easy way to decide if an infinite word is the $\beta$-expansion of a real number [17. For more details, see, for instance, [15, Chap. 7]. First, let us recall the definition of a deterministic finite automaton.

Definition 4. A deterministic finite automaton (DFA), over an alphabet $A$ is given by a 5 -tuple $\mathcal{A}=$ $\left(Q, q_{0}, A, \delta, F\right)$ where $Q$ is a finite set of states, $q_{0} \in Q$ is the initial state, $\delta: Q \times A \mapsto Q$ is the transition function and $F \subset Q$ is the set of final states (graphically represented by two concentric circles). The map $\delta$ can be extended to $Q \times A^{*}$ by setting $\delta(q, \varepsilon)=q$ and $\delta(q, w a)=\delta(\delta(q, w), a)$ for all $q \in Q, a \in A$ and $w \in A^{*}$. We also say that a word $w$ is accepted by the automaton if $\delta\left(q_{0}, w\right) \in F$.

Proposition 5. Let $\beta \in \mathbb{R}_{>1}$ be a Parry number.

(a) Suppose that $d_{\beta}(1)=t_{1} \cdots t_{m}$ is finite, i.e., $t_{m} \neq 0$ and $t_{j}=0$ for all $j>m$. Then an infinite word is the $\beta$-expansion of a real number in $[0,1)$ if and only if it is the label of a path in the automaton $\mathcal{A}_{\beta}=\left(\left\{a_{0}, \ldots, a_{m-1}\right\}, a_{0}, A_{\beta}, \delta,\left\{a_{0}, \ldots, a_{m-1}\right\}\right)$ depicted in Figure $1 a$. 


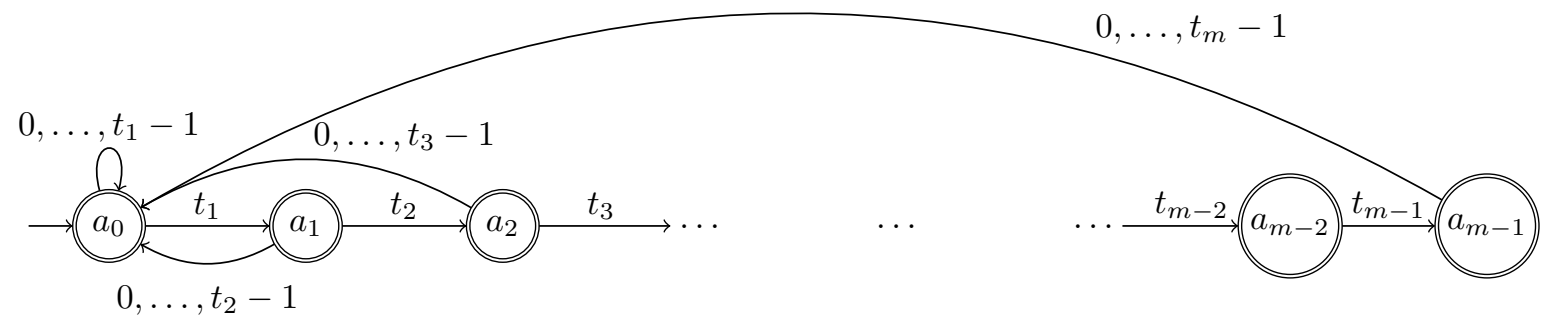

(a) The case when $d_{\beta}(1)$ is finite.

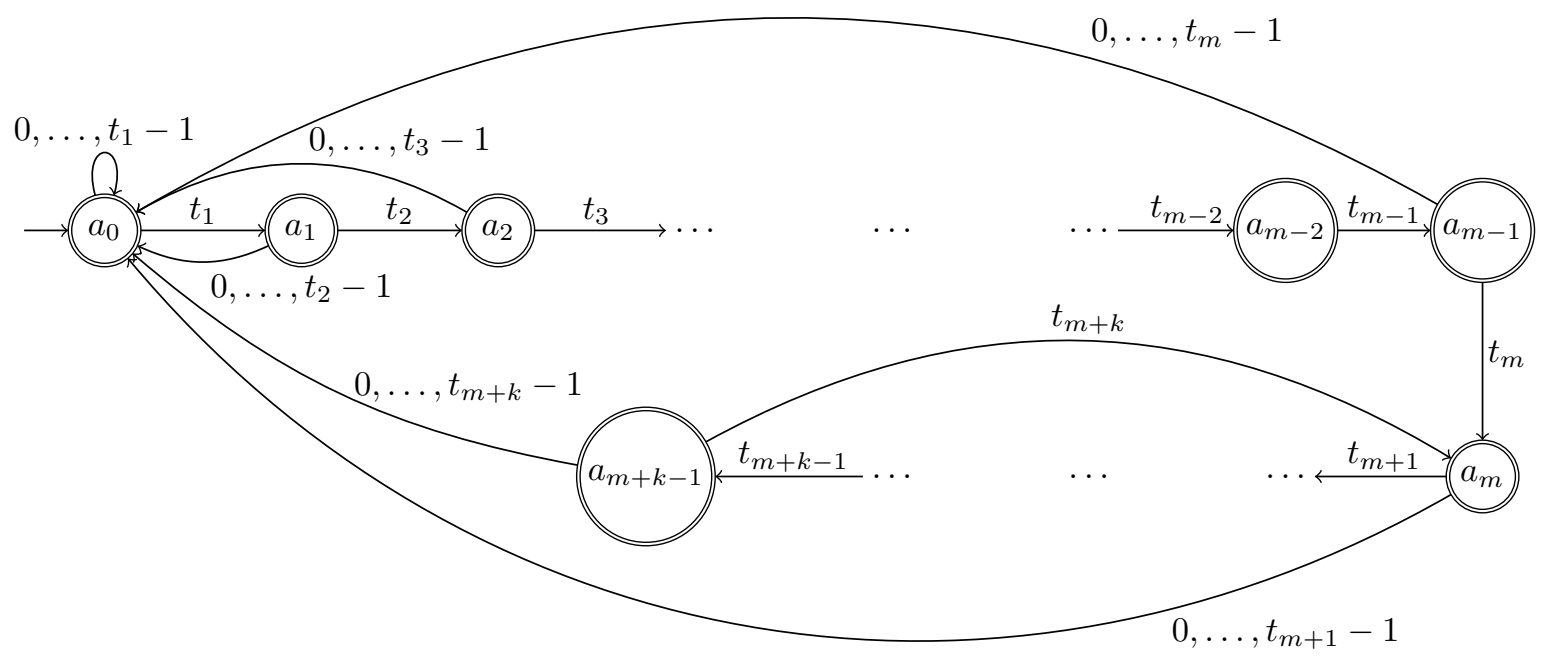

(b) The case when $d_{\beta}(1)$ is ultimately periodic but not finite.

Figure 1: The automaton $\mathcal{A}_{\beta}$ in function of the ultimately periodic word $d_{\beta}(1)$. 


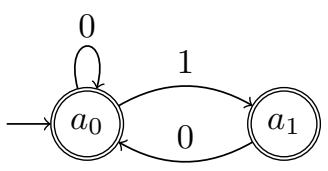

(a)

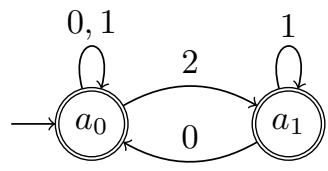

(b)

Figure 2: The automaton $\mathcal{A}_{\varphi}$ (on the left) and the automaton $\mathcal{A}_{\varphi^{2}}$ (on the right).

(b) Suppose that $d_{\beta}(1)=t_{1} \cdots t_{m}\left(t_{m+1} \cdots t_{m+k}\right)^{\omega}$ where $m, k$ are taken to be minimal. Then an infinite word is the $\beta$-expansion of a real number $[0,1)$ if and only if it is the label of a path in the automaton $\mathcal{A}_{\beta}=\left(\left\{a_{0}, \ldots, a_{m+k-1}\right\}, a_{0}, A_{\beta}, \delta,\left\{a_{0}, \ldots, a_{m+k-1}\right\}\right)$ depicted in Figure $1 b$.

Let us illustrate the previous proposition. For other examples, see, for instance, [5].

Example 6. If $\beta \in \mathbb{R}_{>1}$ is an integer, then $d_{\beta}(1)=d_{\beta}^{*}(1)=(\beta-1)^{\omega}$. The automaton $\mathcal{A}_{\beta}$ consists of a single initial and final state $a_{0}$ with a loop of labels $0,1, \ldots, \beta-1$.

Example 7. Consider the golden ratio $\varphi$. Since $1=1 / \varphi+1 / \varphi^{2}$, we have $d_{\varphi}(1)=11$ and $d_{\varphi}^{*}(1)=(10)^{\omega}$. It is thus a Parry number. The automaton $\mathcal{A}_{\varphi}$ is depicted in Figure 2a.

The square $\varphi^{2}$ of the golden ratio is again a Parry number with $d_{\varphi^{2}}(1)=d_{\varphi^{2}}^{*}(1)=21^{\omega}$. The automaton $\mathcal{A}_{\varphi^{2}}$ is depicted in Figure $2 \mathrm{~b}$

With every Parry number is canonically associated a linear numeration system. Let us recall the definition of such numeration systems.

Definition 8. Let $U=(U(n))_{n \geq 0}$ be a sequence of integers such that $U$ is increasing, $U(0)=1$ and $\sup _{n \geq 0} \frac{U(n+1)}{U(n)}$ is bounded by a constant. We say that $U$ is a linear numeration system if $U$ satisfies a linear recurrence relation, i.e., there exist $k \geq 1$ and $a_{0}, \ldots, a_{k-1} \in \mathbb{Z}$ such that

$$
\forall n \geq 0, \quad U(n+k)=a_{k-1} U(n+k-1)+\cdots+a_{0} U(n) .
$$

Let $n$ be a positive integer. By successive Euclidean divisions, there exists $\ell \geq 1$ such that

$$
n=\sum_{j=0}^{\ell-1} c_{j} U(j)
$$

where the $c_{j}$ 's are non-negative integers and $c_{\ell-1}$ is non-zero. The word $c_{\ell-1} \cdots c_{0}$ is called the normal $U$ representation of $n$ and is denoted by $\operatorname{rep}_{U}(n)$. In other words, the word $c_{\ell-1} \cdots c_{0}$ is the greedy expansion of $n$ in the considered numeration system. We set $\operatorname{rep}_{U}(0):=\varepsilon$. Finally, we refer to $L_{U}:=\operatorname{rep}_{U}(\mathbb{N})$ as the language of the numeration and we let $A_{U}$ denote the minimal alphabet such that $L_{U} \subset A_{U}^{*}$. If $d_{r} \cdots d_{0}$ is a word over an alphabet of digits, then its $U$-numerical value is

$$
\operatorname{val}_{U}\left(d_{r} \cdots d_{0}\right):=\sum_{j=0}^{r} d_{j} U(j) .
$$

Observe that, if $\operatorname{val}_{U}\left(d_{r} \cdots d_{0}\right)=n$, then the word $d_{r} \cdots d_{0}$ is a $U$-representation of $n$ (but not necessarily its normal $U$-representation).

Definition 9. Let $\beta \in \mathbb{R}_{>1}$ be a Parry number. We define a particular linear numeration system $U_{\beta}:=$ $\left(U_{\beta}(n)\right)_{n \geq 0}$ associated with $\beta$ as follows.

If $d_{\beta}(1)=t_{1} \cdots t_{m}$ is finite $\left(t_{m} \neq 0\right)$, then we set $U_{\beta}(0):=1, U_{\beta}(i):=t_{1} U_{\beta}(i-1)+\cdots+t_{i} U_{\beta}(0)+1$ for all $i \in\{1, \ldots, m-1\}$ and, for all $n \geq m$,

$$
U_{\beta}(n):=t_{1} U_{\beta}(n-1)+\cdots+t_{m} U_{\beta}(n-m) .
$$

If $d_{\beta}(1)=t_{1} \cdots t_{m}\left(t_{m+1} \cdots t_{m+k}\right)^{\omega}(m, k$ are minimal $)$, then we set $U_{\beta}(0):=1, U_{\beta}(i):=t_{1} U_{\beta}(i-1)+$ $\cdots+t_{i} U_{\beta}(0)+1$ for all $i \in\{1, \ldots, m+k-1\}$ and, for all $n \geq m+k$,

$$
\begin{aligned}
U_{\beta}(n):= & t_{1} U_{\beta}(n-1)+\cdots+t_{m+k} U_{\beta}(n-m-k)+U_{\beta}(n-k) \\
& -t_{1} U_{\beta}(n-k-1)-\cdots-t_{m} U_{\beta}(n-m-k) .
\end{aligned}
$$


The linear numeration system $U_{\beta}$ from Definition 9 has an interesting property: it is a Bertrand numeration system.

Definition 10. A linear numeration system $U=(U(n))_{n \geq 0}$ is a Bertrand numeration system if, for all $w \in A_{U}^{+}, w \in L_{U} \Leftrightarrow w 0 \in L_{U}$.

Bertrand proved that the linear numeration system $U_{\beta}$ associated with the Parry number $\beta$ from Definition 9 is the unique linear numeration system associated with $\beta$ that is also a Bertrand numeration system [4. In that case [4], any word $w$ in the set $0^{*} L_{U_{\beta}}$ of all normal $U_{\beta}$-representations with leading zeroes is the label of a path in the automaton $\mathcal{A}_{\beta}$ from Proposition 5

Finally, every Parry number is a Perron number [15, Chap. 7]. A real number $\beta>1$ is a Perron number if it is an algebraic integer whose conjugates have modulus less than $\beta$. Numeration systems based on Perron numbers are defined as follows and have the property (2), which is often used in this paper.

Definition 11. Let $U=(U(n))_{n \geq 0}$ be a linear numeration system. Consider the characteristic polynomial of the recurrence (1) given by $P(X)=X^{k}-a_{k-1} X^{k-1}-\cdots-a_{1} X-a_{0}$. If $P$ is the minimal polynomial of a Perron number $\beta \in \mathbb{R}_{>1}$, we say that $U$ is a Perron numeration system. In this case, the polynomial $P$ can be factored as

$$
P(X)=(X-\beta)\left(X-\alpha_{2}\right) \cdots\left(X-\alpha_{k}\right)
$$

where the complex numbers $\alpha_{2}, \ldots, \alpha_{k}$ are the conjugates of $\beta$, and, for all $j>1$, we have $\left|\alpha_{j}\right|<\beta$. Using a well-known fact regarding recurrence relations, we have

$$
U(n)=c_{1} \beta^{n}+c_{2} \alpha_{2}^{n}+\cdots+c_{k} \alpha_{k}^{n} \quad \forall n \geq 0
$$

where $c_{1}, \ldots, c_{k}$ are complex numbers depending on the initial values of $U$. Since $\left|\alpha_{j}\right|<\beta$ for all $j>1$, we have

$$
\lim _{n \rightarrow+\infty} \frac{U(n)}{\beta^{n}}=c_{1}
$$

Remark 12. Note that if two Perron numeration systems are associated with the same Perron number, then these two systems only differ by the choice of the initial values $U(0), \ldots, U(k-1)$. The choice of those initial values is of great importance. See, for instance, Example 14.

Example 13. The usual integer base system is a special case of a Perron-Bertrand numeration system.

Example 14. The golden ratio $\varphi$ is a Perron number whose minimal polynomial is $P(X)=X^{2}-X-1$. A Perron-Bertrand numeration system associated with $\varphi$ is the Fibonacci numeration system based on the Fibonacci numbers $(F(n))_{n \geq 0}$ defined by $F(0)=1, F(1)=2$ and $F(n+2)=F(n+1)+F(n)$. If we change the initial conditions and set $F^{\prime}(0)=1, F^{\prime}(1)=3$ and $F^{\prime}(n+2)=F^{\prime}(n+1)+F^{\prime}(n)$, we again get a Perron numeration associated with $\varphi$ which is not a Bertrand numeration system. Indeed, 2 is a greedy representation, but not 20 because $\operatorname{rep}_{F^{\prime}}\left(\operatorname{val}_{F^{\prime}}(20)\right)=102$.

The particular setting of this paper is the following one: we let $\beta \in \mathbb{R}_{>1}$ be a Parry number and we constantly use the special Parry-Bertrand numeration $U_{\beta}$ from Definition 9. From Definition 3 and Definition 8 the alphabet $A_{U_{\beta}}$ is the set $\{0,1, \ldots,\lceil\beta\rceil-1\}$ and the language of the system of numeration $U_{\beta}$ is $L_{U_{\beta}} \subset A_{U_{\beta}}^{*}$ (which is defined using the automaton $\mathcal{A}_{\beta}$ from Proposition 5). To end this section, we prove a useful lemma about binomial coefficients of words ending with blocks of zeroes.

Lemma 15. For all non-empty words $u, v \in L_{U_{\beta}}$ and all $k \in \mathbb{N}$, we have

$$
\left(\begin{array}{l}
u 0^{k} \\
v 0^{k}
\end{array}\right)=\sum_{j=0}^{k}\left(\begin{array}{l}
k \\
j
\end{array}\right)\left(\begin{array}{c}
u \\
v 0^{j}
\end{array}\right) .
$$


Proof. We proceed by induction on $k \in \mathbb{N}$. If $k=0$, the result is obvious. Suppose that the result holds true for all non-empty words $u, v \in L_{U_{\beta}}$ and for $0, \ldots, k$. We show that it still holds true for all non-empty words $u, v \in L_{U_{\beta}}$ and $k+1$. Using Lemma 11, we first have

$$
\left(\begin{array}{l}
u 0^{k+1} \\
v 0^{k+1}
\end{array}\right)=\left(\begin{array}{c}
u 0^{k} \\
v^{\prime} 0^{k}
\end{array}\right)+\left(\begin{array}{l}
u 0^{k} \\
v 0^{k}
\end{array}\right)
$$

where $v^{\prime}=v 0 \in L_{U_{\beta}}$ since $U_{\beta}$ is a Parry-Bertrand numeration system. By induction hypothesis, we get

$$
\begin{aligned}
\left(\begin{array}{c}
u 0^{k+1} \\
v 0^{k+1}
\end{array}\right) & =\sum_{j=1}^{k+1}\left(\begin{array}{c}
k \\
j-1
\end{array}\right)\left(\begin{array}{c}
u \\
v 0^{j}
\end{array}\right)+\sum_{j=0}^{k}\left(\begin{array}{c}
k \\
j
\end{array}\right)\left(\begin{array}{c}
u \\
v 0^{j}
\end{array}\right) \\
& =\left(\begin{array}{c}
k+1 \\
k+1
\end{array}\right)\left(\begin{array}{c}
u \\
v 0^{k+1}
\end{array}\right)+\sum_{j=1}^{k}\left(\left(\begin{array}{c}
k \\
j-1
\end{array}\right)+\left(\begin{array}{c}
k \\
j
\end{array}\right)\right)\left(\begin{array}{c}
u \\
v 0^{j}
\end{array}\right)+\left(\begin{array}{c}
k+1 \\
0
\end{array}\right)\left(\begin{array}{l}
u \\
v
\end{array}\right) \\
& =\sum_{j=0}^{k+1}\left(\begin{array}{c}
k+1 \\
j
\end{array}\right)\left(\begin{array}{c}
u \\
v 0^{j}
\end{array}\right) .
\end{aligned}
$$

\section{The $(\star)$ condition}

We let $w_{n}=\operatorname{rep}_{U_{\beta}}(n)$ denote the $n$th word of the language $L_{U_{\beta}}$ in the genealogical order. The generalized Pascal triangle $\mathrm{P}_{U_{\beta}}: \mathbb{N} \times \mathbb{N} \rightarrow \mathbb{N}:(i, j) \mapsto\left(\begin{array}{c}w_{i} \\ w_{j}\end{array}\right)$ is represented as an infinite table ${ }^{3}$ whose entry on the $i$ th row and the $j$ th column is the binomial coefficient $\left(\begin{array}{c}w_{i} \\ w_{i}\end{array}\right)$. For instance, when $\beta=\varphi$, the first few values in the generalized Pascal triangle $\mathrm{P}_{U_{\varphi}}$ are given in Table 2 below. Considering the intersection of the lattice $\mathbb{N}^{2}$ with $\left[0, U_{\beta}(n)\right] \times\left[0, U_{\beta}(n)\right]$, the first $U_{\beta}(n)$ rows and columns of the generalized Pascal triangle $\mathrm{P}_{U_{\beta}}$ modulo 2

$$
\left(\left(\begin{array}{c}
w_{i} \\
w_{j}
\end{array}\right) \bmod 2\right)_{0 \leq i, j<U_{\beta}(n)}
$$

provide a coloring of this lattice, leading to a sequence of compact subsets of $\mathbb{R}^{2}$. If we normalize these sets respectively by a homothety of ratio $1 / U_{\beta}(n)$, we define a sequence $\left(\mathcal{U}_{n}^{\beta}\right)_{n \geq 0}$ of subsets of $[0,1] \times[0,1]$.

Definition 16. Let $Q:=[0,1] \times[0,1]$. Consider the sequence $\left(\mathcal{U}_{n}^{\beta}\right)_{n \geq 0}$ of sets in $[0,1] \times[0,1]$ defined for all $n \geq 0$ by

$$
\mathcal{U}_{n}^{\beta}:=\frac{1}{U_{\beta}(n)} \bigcup\left\{\left(\operatorname{val}_{U_{\beta}}(v), \operatorname{val}_{U_{\beta}}(u)\right)+Q \mid u, v \in L_{U_{\beta}},\left(\begin{array}{l}
u \\
v
\end{array}\right) \equiv 1 \bmod 2\right\} \subset[0,1] \times[0,1] .
$$

Each $\mathcal{U}_{n}^{\beta}$ is a finite union of squares of size $1 / U_{\beta}(n)$ and is thus compact.

Example 17. When $\beta=\varphi$ is the golden ratio, the first values in the generalized Pascal triangle $\mathrm{P}_{U_{\varphi}}$ are given in Table 2. The sets $\mathcal{U}_{3}^{\varphi}, \mathcal{U}_{4}^{\varphi}$ and $\mathcal{U}_{5}^{\varphi}$ are depicted in Figure 3 . The set $\mathcal{U}_{9}^{\varphi}$ is depicted in Figure 14 given in the appendix.

Remark 18. Each pair $(u, v)$ of words of length at most $n$ with an odd binomial coefficient gives rise to a square region in $\mathcal{U}_{n}^{\beta}$. More precisely, we have the following situation. Let $n \geq 0$ and $u, v \in L_{U_{\beta}}$ such that $0 \leq|v| \leq|u| \leq n$ and $\left(\begin{array}{l}u \\ v\end{array}\right) \equiv 1 \bmod 2$. We have

$$
\left(\left(\operatorname{val}_{U_{\beta}}(v), \operatorname{val}_{U_{\beta}}(u)\right)+Q\right) / U_{\beta}(n) \subset \mathcal{U}_{n}^{\beta}
$$




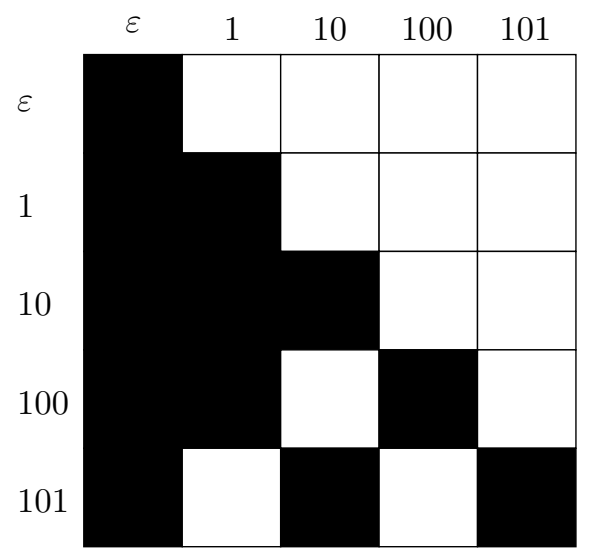

(a) The $\operatorname{set} \mathcal{U}_{3}^{\varphi}$.

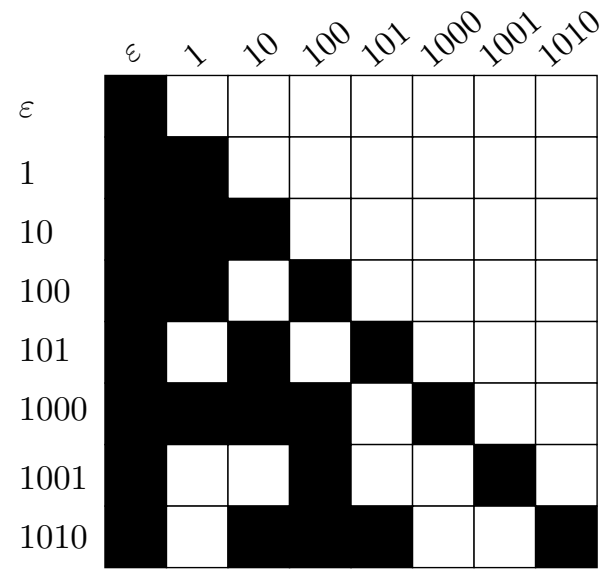

(b) The set $\mathcal{U}_{4}^{\varphi}$.

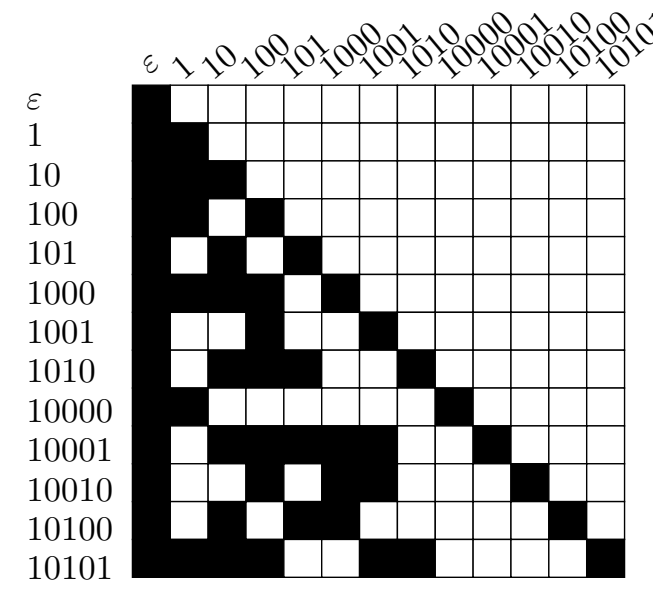

(c) The set $\mathcal{U}_{5}^{\varphi}$.

Figure 3: The sets $\mathcal{U}_{3}^{\varphi}, \mathcal{U}_{4}^{\varphi}$ and $\mathcal{U}_{5}^{\varphi}$ when $\beta=\varphi$ is the golden ratio. 


\begin{tabular}{|c|c|c|c|c|c|c|c|c|c|}
\hline & $\varepsilon$ & 1 & 10 & 100 & $\begin{array}{c}j \\
101\end{array}$ & 1000 & 1001 & 1010 \\
\hline & $\varepsilon$ & 1 & 0 & 0 & 0 & 0 & 0 & 0 & 0 \\
\hline & 1 & 1 & 1 & 0 & 0 & 0 & 0 & 0 & 0 \\
\hline & 10 & 1 & 1 & 1 & 0 & 0 & 0 & 0 & 0 \\
\hline$i$ & 100 & 1 & 1 & 2 & 1 & 0 & 0 & 0 & 0 \\
\hline & 101 & 1 & 2 & 1 & 0 & 1 & 0 & 0 & 0 \\
\hline & 1000 & 1 & 1 & 3 & 3 & 0 & 1 & 0 & 0 \\
\hline & 1001 & 1 & 2 & 2 & 1 & 2 & 0 & 1 & 0 \\
\hline & 1010 & 1 & 2 & 3 & 1 & 1 & 0 & 0 & 1 \\
\hline
\end{tabular}

Table 2: The first few values in the generalized Pascal triangle $\mathrm{P}_{U_{\varphi}}$.

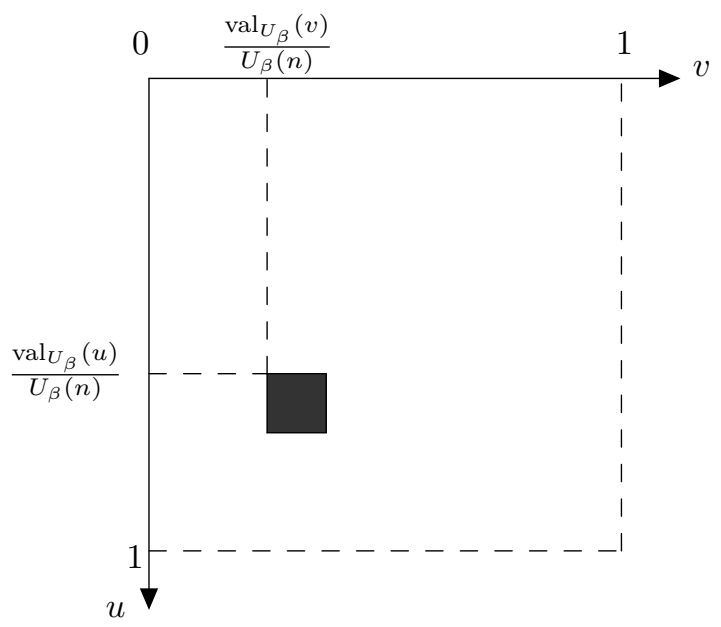

Figure 4: Visualization of a square region in $\mathcal{U}_{n}^{\beta}$.

as depicted in Figure 4

We consider the space $\left(\mathcal{H}\left(\mathbb{R}^{2}\right), d_{h}\right)$ of the non-empty compact subsets of $\mathbb{R}^{2}$ equipped with the Hausdorff metric $d_{h}$ induced by the Euclidean distance $d$ on $\mathbb{R}^{2}$. It is well known that $\left(\mathcal{H}\left(\mathbb{R}^{2}\right), d_{h}\right)$ is complete $[\underline{6}$. We let $B(x, \epsilon)$ denote the open ball of radius $\epsilon \geq 0$ centered at $x \in \mathbb{R}^{2}$ and, if $S \subset \mathbb{R}^{2}$, we let

$$
[S]_{\epsilon}:=\bigcup_{x \in S} B(x, \epsilon)
$$

denote $\epsilon$-fattening of $S$.

Our aim is to show that the sequence $\left(\mathcal{U}_{n}^{\beta}\right)_{n>0}$ of compact subsets of $[0,1] \times[0,1]$ is converging and to provide an elementary description of its limit set. The idea is the following one. Let $(u, v) \in L_{U_{\beta}} \times L_{U_{\beta}}$ be a pair of words having an odd binomial coefficient. On the one hand, some of those pairs are such that $\left(\begin{array}{c}u a \\ v a\end{array}\right) \equiv 0 \bmod 2$ for all letters $a$ such that $u a, v a \in L_{U_{\beta}}$. In other words, those pairs of words create a black square region in $\mathcal{U}_{|u|}^{\beta}$ while the corresponding square region in $\mathcal{U}_{|u|+1}^{\beta}$ is white. As an example, take $\beta=\varphi, u=1010$ and $v=101$. We have $\left(\begin{array}{l}u 0 \\ v 0\end{array}\right)=2$ (see Figure 3). On the other hand, some of those pairs create a more stable pattern, i.e., $\left(\begin{array}{l}u w \\ v w\end{array}\right) \equiv 1 \bmod 2$ for all words $w$ such that $u w, v w \in L_{U_{\beta}}$. Roughly, those

\footnotetext{
${ }^{3}$ Using the notation $\left(\begin{array}{l}u \\ v\end{array}\right)$, the rows (resp., columns) of $\mathrm{P}_{U_{\beta}}$ are indexed by the words $u$ (resp., $v$ ).
} 


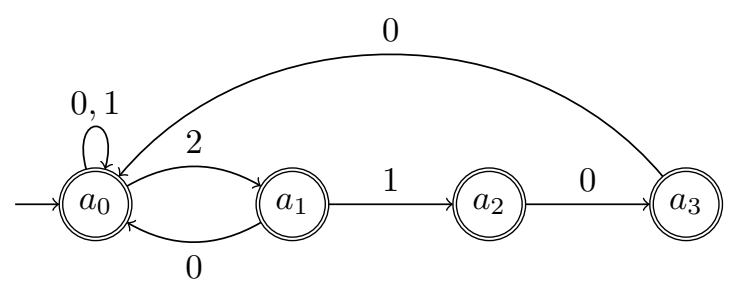

Figure 5: The automaton $\mathcal{A}_{\beta}$ for the dominant root $\beta$ of the polynomial $P(X)=X^{4}-2 X^{3}-X^{2}-1$.

pairs create a diagonal of square regions in $\left(\mathcal{U}_{n}^{\beta}\right)_{n \geq 0}$. For instance, take $\beta=\varphi, u=101$ and $v=10$. In this case, $\left(\begin{array}{c}u w \\ v w\end{array}\right) \equiv 1 \bmod 2$ for all admissible words $w$. In particular, the pairs of words $(u, v),(u 0, v 0)$ and $(u 00, v 00),(u 01, v 01)$ have odd binomial coefficients (see Figure 3$)$ and create a diagonal of square regions. With the second type of pairs of words, we define a new sequence of compact subsets $\left(\mathcal{A}_{n}^{\beta}\right)_{n \geq 0}$ of $[0,1] \times[0,1]$ which converges to some well-defined limit set $\mathcal{L}^{\beta}$. Then, we show that the first sequence of compact sets $\left(\mathcal{U}_{n}^{\beta}\right)_{n \geq 0}$ also converges to this limit set. The remaining of this paper is dedicated to formalize and prove those statements.

To reach that goal, for all non-empty words $u, v \in L_{U_{\beta}}$, we first define the least integer $p$ such that $u 0^{p} w, v 0^{p} w$ belong to $L_{U_{\beta}}$ for all words $w \in 0^{*} L_{U_{\beta}}$. In other terms, any word $w$ can be read after $u 0^{p}$ and $v 0^{p}$ in the automaton $\mathcal{A}_{\beta}$. Then, some pairs of words $(u, v) \in L_{U_{\beta}} \times L_{U_{\beta}}$ have the property that not only $\left(\begin{array}{l}u \\ v\end{array}\right) \equiv 1 \bmod 2$ but also $\left(\begin{array}{l}u 0^{p} w \\ v 0^{p} w\end{array}\right) \equiv 1 \bmod 2$ for all words $w \in 0^{*} L_{U_{\beta}}$; see Corollary 28 . Such a property creates a particular pattern occurring in $\mathcal{U}_{n}^{\beta}$ for all sufficiently large $n$, as shown in Remark 30.

Proposition 19. For all non-empty words $u, v \in L_{U_{\beta}}$, there exists a smallest nonnegative integer $p(u, v)$ such that

$$
\left(u 0^{p(u, v)}\right)^{-1} \cdot L_{U_{\beta}}=\left(v 0^{p(u, v)}\right)^{-1} \cdot L_{U_{\beta}}=0^{*} L_{U_{\beta}} .
$$

Proof. Using Proposition 5, take $p(u, v)$ to be the least nonnegative integer $p$ such that $\delta\left(a_{0}, u 0^{p}\right)=a_{0}=$ $\delta\left(a_{0}, v 0^{p}\right)$. Then, for any word $w \in 0^{*} L_{U_{\beta}}$, the words $u 0^{p(u, v)} w, v 0^{p(u, v)} w$ are labels of paths in $\mathcal{A}_{\beta}$. Consequently, they are words in $L_{U_{\beta}}$. Conversely, if the words $u 0^{p(u, v)} w, v 0^{p(u, v)} w$ are labels of paths in $\mathcal{A}_{\beta}$, then $w \in 0^{*} L_{U_{\beta}}$.

In the following, we will be using $p(\varepsilon, \varepsilon)$. Observe that, using Proposition $5, \delta\left(a_{0}, \varepsilon\right)=a_{0}$. We naturally set $p(\varepsilon, \varepsilon):=0$ and we thus have $\left(\varepsilon 0^{p(\varepsilon, \varepsilon)}\right)^{-1} \cdot L_{U_{\beta}}=L_{U_{\beta}}$.

Example 20. If $\beta>1$ is an integer, then $p(u, v)=0$ for all $u, v \in L_{U_{\beta}}$. See Example 6

Example 21. If $\beta=\varphi$ is the golden ratio, then $p(u, v)=0$ if and only if $u$ and $v$ end with 0 or $u=v=\varepsilon$, otherwise $p(u, v)=1$.

The integer of Proposition 19 can be greater than 1 as illustrated in the following example.

Example 22. Let $\beta$ be the dominant root of the polynomial $P(X)=X^{4}-2 X^{3}-X^{2}-1$. Then $\beta \approx 2.47098$ is a Parry number with $d_{\beta}(1)=2101$ and $d_{\beta}^{*}(1)=(2100)^{\omega}$. The automaton $\mathcal{A}_{\beta}$ is depicted in Figure 5 . For instance, $p(101,21)=2$.

Definition 23. Let $(u, v) \in L_{U_{\beta}} \times L_{U_{\beta}}$. We say that $(u, v)$ satisfies the $(\star)$ condition if either $u=v=\varepsilon$, or $|u| \geq|v|>0$ and

$$
\left(\begin{array}{c}
u 0^{p(u, v)} \\
v 0^{p(u, v)}
\end{array}\right) \equiv 1 \bmod 2 \quad \text { and } \quad\left(\begin{array}{c}
u 0^{p(u, v)} \\
v 0^{p(u, v)} a
\end{array}\right)=0 \quad \forall a \in A_{U_{\beta}}
$$

where $p(u, v)$ is defined by Proposition 19 Observe that, if $(u, v) \neq(\varepsilon, \varepsilon)$, then $v 0^{p(u, v)} a \in L_{U_{\beta}}$ for all $a \in A_{U_{\beta}}$. 
Remark 24. Observe that if only one of the two words $u$ or $v$ is empty, then the pair $(u, v)$ never satisfies $(\star)$.

The next lemma shows that all diagonal elements of $\mathcal{U}_{n}^{\beta}$ satisfy $(\star)$.

Lemma 25. For any word $u \in L_{U_{\beta}}$, the pair $(u, u)$ satisfies $(\star)$.

Proof. If $u=\varepsilon$, the result is clear using Definition 23. Suppose $u$ is non-empty and let $p:=p(u, u)$ denote the integer from Proposition 19 . Then, for all $a \in A_{U_{\beta}}$, we have

$$
\left(\begin{array}{c}
u 0^{p} \\
u 0^{p}
\end{array}\right)=1 \equiv 1 \bmod 2 \quad \text { and } \quad\left(\begin{array}{c}
u 0^{p} \\
u 0^{p} a
\end{array}\right)=0
$$

since $\left|u 0^{p} a\right|>\left|u 0^{p}\right|$.

If a pair of words satisfies $(\star)$, it has the following two properties. First, its binomial coefficient is odd, as stated in the following proposition. Secondly, it creates a special pattern in $\mathcal{U}_{n}^{\beta}$ for all large enough $n$; see Proposition 27, Corollary 28 and Remark 30.

Proposition 26. Let $(u, v) \in L_{U_{\beta}} \times L_{U_{\beta}}$ satisfying $(\star)$. Then

$$
\left(\begin{array}{l}
u \\
v
\end{array}\right) \equiv 1 \bmod 2 .
$$

Proof. If $u=v=\varepsilon$, the result is clear by definition. Suppose that $u$ and $v$ are non-empty. Let us proceed by contradiction and suppose that $\left(\begin{array}{l}u \\ v\end{array}\right)$ is even. Let us set $p:=p(u, v)$ from Proposition 19. On the one hand, by Definition 23 , we know that

$$
\left(\begin{array}{c}
u 0^{p} \\
v 0^{p}
\end{array}\right) \equiv 1 \bmod 2
$$

and, on the other hand, Lemma 15 states that

$$
\left(\begin{array}{l}
u 0^{p} \\
v 0^{p}
\end{array}\right)=\sum_{j=1}^{p}\left(\begin{array}{c}
p \\
j
\end{array}\right)\left(\begin{array}{c}
u \\
v 0^{j}
\end{array}\right)+\left(\begin{array}{l}
u \\
v
\end{array}\right)
$$

Consequently, we have

$$
\sum_{j=1}^{p}\left(\begin{array}{l}
p \\
j
\end{array}\right)\left(\begin{array}{c}
u \\
v 0^{j}
\end{array}\right) \equiv 1 \bmod 2>0
$$

and there must exist $i \in\{1, \ldots, p\}$ such that $\left(\begin{array}{c}u \\ v 0^{i}\end{array}\right)>0$. Using again Lemma 15 we also have

$$
\left(\begin{array}{c}
u 0^{p} \\
v 0^{p} 0
\end{array}\right)=\sum_{j=1}^{p+1}\left(\begin{array}{c}
p \\
j-1
\end{array}\right)\left(\begin{array}{c}
u \\
v 0^{j}
\end{array}\right) \geq\left(\begin{array}{c}
p \\
i-1
\end{array}\right)\left(\begin{array}{c}
u \\
v 0^{i}
\end{array}\right)>0,
$$

which contradicts Definition 23.

Proposition 27. Let $u, v \in L_{U_{\beta}}$ be two non-empty words such that $(u, v)$ satisfies $(\star)$. For any letter $a \in A_{U_{\beta}}$, the pair of words $\left(u 0^{p(u, v)} a, v 0^{p(u, v)} a\right) \in L_{U_{\beta}} \times L_{U_{\beta}}$ satisfies $(\star)$.

Proof. For the sake of clarity, set $p:=p(u, v)$. Let $a$ be a letter in $A_{U_{\beta}}$ and also set $p^{\prime}:=p\left(u 0^{p} a, v 0^{p} a\right)$. By Lemma 1 and Lemma 15

$$
\left(\begin{array}{c}
u 0^{p} a 0^{p^{\prime}} \\
v 0^{p} a 0^{p^{\prime}}
\end{array}\right)=\sum_{j=1}^{p^{\prime}}\left(\begin{array}{c}
p^{\prime} \\
j
\end{array}\right)\left(\begin{array}{c}
u 0^{p} a \\
v 0^{p} a 0^{j}
\end{array}\right)+\left(\begin{array}{c}
u 0^{p} \\
v 0^{p} a
\end{array}\right)+\left(\begin{array}{c}
u 0^{p} \\
v 0^{p}
\end{array}\right) .
$$


Since $(u, v)$ satisfies $(\star)$, all the coefficients $\left(\begin{array}{c}u 0^{p} a \\ v 0^{p} a 0^{j}\end{array}\right)$, for $j=1, \ldots, p^{\prime}$, and $\left(\begin{array}{c}u 0^{p} \\ v 0^{p} a\end{array}\right)$ are equal to 0 . Otherwise, it means that the word $v 0^{p} a$ appears as a subword of the word $u 0^{p}$, which contradicts $(\star)$. Consequently, using Definition 23, we get

$$
\left(\begin{array}{l}
u 0^{p} a 0^{p^{\prime}} \\
v 0^{p} a 0^{p^{\prime}}
\end{array}\right)=\left(\begin{array}{l}
u 0^{p} \\
v 0^{p}
\end{array}\right) \equiv 1 \bmod 2 .
$$

Using the same argument, for any letter $b \in A_{U_{\beta}}$, we have

$$
\left(\begin{array}{c}
u 0^{p} a 0^{p^{\prime}} \\
v 0^{p} a 0^{p^{\prime}} b
\end{array}\right)=0
$$

The next corollary extends Lemma 25 when $(u, v) \neq(\varepsilon, \varepsilon)$. Indeed, recall that $p(\varepsilon, \varepsilon)=0$.

Corollary 28. Let $u, v \in L_{U_{\beta}}$ be two non-empty words such that $(u, v)$ satisfies $(\star)$. Then

$$
\left(\begin{array}{c}
u 0^{p(u, v)} w \\
v 0^{p(u, v)} w
\end{array}\right) \equiv 1 \bmod 2 \quad \forall w \in 0^{*} L_{U_{\beta}} .
$$

Proof. Set $p:=p(u, v)$. From Proposition 19, $u 0^{p} w, v 0^{p} w$ belong to $L_{U_{\beta}}$ for any word $w \in 0^{*} L_{U_{\beta}}$. Now proceed by induction on the length of $w \in 0^{*} L_{U_{\beta}}$. If $|w|=0$, then $w=\varepsilon$ is the empty word and the statement is true using Definition 23. If $|w|=1$, then $w=a$ is a letter belonging to $A_{U_{\beta}}$. Then, by Proposition 27, we know that $\left(u 0^{p} a, v 0^{p} a\right)$ satisfies $(\star)$. Using Proposition 26 , we have

$$
\left(\begin{array}{l}
u 0^{p} a \\
v 0^{p} a
\end{array}\right) \equiv 1 \bmod 2 .
$$

Now suppose that $|w| \geq 2$ and write $w=a w^{\prime} b \in 0^{*} L_{U_{\beta}}$ where $a, b$ are letters. From Lemma 1 we deduce that

$$
\left(\begin{array}{c}
u 0^{p} w \\
v 0^{p} w
\end{array}\right)=\left(\begin{array}{c}
u 0^{p} a w^{\prime} \\
v 0^{p} a w^{\prime} b
\end{array}\right)+\left(\begin{array}{c}
u 0^{p} a w^{\prime} \\
v 0^{p} a w^{\prime}
\end{array}\right) .
$$

By induction hypothesis, $\left(\begin{array}{c}u 0^{p} a w^{\prime} \\ v 0^{p} a w^{\prime}\end{array}\right) \equiv 1 \bmod 2$ since $a w^{\prime} \in 0^{*} L_{U_{\beta}}$ and $\left|a w^{\prime}\right|<|w|$. Furthermore, $\left(\begin{array}{l}u 0^{p} a w^{\prime} \\ v 0^{p} a w^{\prime} b\end{array}\right)$ must be 0 , otherwise it means that the word $v 0^{p} a$ occurs as a subword of the word $u 0^{p}$, which contradicts the fact that $(u, v)$ satisfies $(\star)$. This ends the proof.

The next lemma is useful to characterize the pattern created in $\mathcal{U}_{n}^{\beta}$, for all sufficiently large $n$, by pairs of words satisfying $(\star)$, see Remark 30 . In this result, we make use of the convention given in Definition 3 .

Lemma 29. Let $(u, v) \in L_{U_{\beta}} \times L_{U_{\beta}}$ satisfying $(\star)$.

(a) The sequence

$$
\left(\left(\frac{\operatorname{val}_{U_{\beta}}\left(v 0^{p(u, v)+n}\right)}{U_{\beta}(|u|+p(u, v)+n)}, \frac{\operatorname{val}_{U_{\beta}}\left(u 0^{p(u, v)+n}\right)}{U_{\beta}(|u|+p(u, v)+n)}\right)\right)_{n \geq 0}
$$

converges to the pair of real numbers $\left(0.0^{|u|-|v|} v, 0 . u\right)$.

(b) For all $n \geq 0$, let $w=d_{n}$ denotes the prefix of length $n$ of $d_{\beta}^{*}(1)$. Then the sequence

$$
\left(\left(\frac{\operatorname{val}_{U_{\beta}}\left(v 0^{p(u, v)} d_{n}\right)}{U_{\beta}(|u|+p(u, v)+n)}, \frac{\operatorname{val}_{U_{\beta}}\left(u 0^{p(u, v)} d_{n}\right)}{U_{\beta}(|u|+p(u, v)+n)}\right)\right)_{n \geq 0}
$$

converges to the pair of real numbers $\left(0.0^{|u|-|v|} v 0^{p(u, v)} d_{\beta}^{*}(1), 0 . u 0^{p(u, v)} d_{\beta}^{*}(1)\right)$. 
Proof. Let $(u, v) \in L_{U_{\beta}} \times L_{U_{\beta}}$ satisfying $(\star)$ and set $p:=p(u, v)$. We prove the first item as the proof of the second one is similar. The result is trivial if $u=v=\varepsilon$. Suppose that $u$ and $v$ are non-empty words. Let us write $u=u_{|u|-1} u_{|u|-2} \cdots u_{0}$ where $u_{i} \in A_{U_{\beta}}$ for all $i$. By definition, we have

$$
\frac{\operatorname{val}_{U_{\beta}}\left(u 0^{p+n}\right)}{U_{\beta}(|u|+p+n)}=\sum_{i=0}^{|u|-1} u_{i} \frac{U_{\beta}(i+p+n)}{U_{\beta}(|u|+p+n)} .
$$

Using (2), $U_{\beta}(i+p+n) / U_{\beta}(|u|+p+n)$ tends to $\beta^{i} / \beta^{|u|}$ when $n$ tend to infinity. Consequently,

$$
\lim _{n \rightarrow+\infty} \frac{\operatorname{val}_{U_{\beta}}\left(u 0^{p+n}\right)}{U_{\beta}(|u|+p+n)}=\sum_{i=0}^{|u|-1} u_{i} \beta^{i-|u|}=0 . u .
$$

Using the same reasoning on the word $v$, we conclude that the sequence

$$
\left(\left(\frac{\operatorname{val}_{U_{\beta}}\left(v 0^{p(u, v)+n}\right)}{U_{\beta}(|u|+p(u, v)+n)}, \frac{\operatorname{val}_{U_{\beta}}\left(u 0^{p(u, v)+n}\right)}{U_{\beta}(|u|+p(u, v)+n)}\right)\right)_{n \geq 0}
$$

converges to the pair of real numbers $\left(0.0^{|u|-|v|} v, 0 . u\right)$.

Remark 30. Let $(u, v) \in L_{U_{\beta}} \times L_{U_{\beta}}$ satisfying $(\star)$ and set $p:=p(u, v)$. Suppose that $u$ and $v$ are nonempty (the case when $u=v=\varepsilon$ is similar: in the following, replace $0^{*} L_{U_{\beta}}$ by $L_{U_{\beta}}$ where needed). Using Corollary 28, the pair of words $\left(u 0^{p} w, v 0^{p} w\right)$ has an odd binomial coefficient for any word $w \in 0^{*} L_{U_{\beta}}$. In particular, the pair of words $\left(u 0^{p} w, v 0^{p} w\right)$ corresponds to a square region in $\mathcal{U}_{|u|+p+n}^{\beta}$ for all $w \in 0^{*} L_{U_{\beta}}$ such that $|w|=n \geq 0$. Using Remark 18 , this region is

$$
\left(\frac{\operatorname{val}_{U_{\beta}}\left(v 0^{p} w\right)}{U_{\beta}(|u|+p+n)}, \frac{\operatorname{val}_{U_{\beta}}\left(u 0^{p} w\right)}{U_{\beta}(|u|+p+n)}\right)+\frac{Q}{U_{\beta}(|u|+p+n)} \subset \mathcal{U}_{|u|+p+n}^{\beta} .
$$

Using Lemma 29, when $w=0^{n}$ (the smallest word of length $n$ in $0^{*} L_{U_{\beta}}$ ), the sequence

$$
\left(\left(\frac{\operatorname{val}_{U_{\beta}}\left(v 0^{p+n}\right)}{U_{\beta}(|u|+p+n)}, \frac{\operatorname{val}_{U_{\beta}}\left(u 0^{p+n}\right)}{U_{\beta}(|u|+p+n)}\right)\right)_{n \geq 0}
$$

converges to the pair of real numbers $\left(0.0^{|u|-|v|} v, 0 . u\right)$. This point will be the first endpoint of a segment associated with $u$ and $v$. See Definition 32. Analogously, using Lemma 29, when $w=d_{n}$ is the prefix of length $n$ of $d_{\beta}^{*}(1)$ (the greatest word of length $n$ in $0^{*} L_{U_{\beta}}$ ), then the sequence

$$
\left(\left(\frac{\operatorname{val}_{U_{\beta}}\left(v 0^{p} d_{n}\right)}{U_{\beta}(|u|+p+n)}, \frac{\operatorname{val}_{U_{\beta}}\left(u 0^{p} d_{n}\right)}{U_{\beta}(|u|+p+n)}\right)\right)_{n \geq 0}
$$

converges to the pair of real numbers $\left(0.0^{|u|-|v|} v 0^{p} d_{\beta}^{*}(1), 0 . u 0^{p} d_{\beta}^{*}(1)\right)$. This point will be the second endpoint of the same segment associated with $u$ and $v$. See Definition 32. As a consequence, the sequence of sets whose $n$th term is defined by

$$
\bigcup_{\substack{|w|=n \\ w \in 0^{*} L_{U_{\beta}}}}\left(\left(\frac{\operatorname{val}_{U_{\beta}}\left(v 0^{p} w\right)}{U_{\beta}(|u|+p+n)}, \frac{\operatorname{val}_{U_{\beta}}\left(u 0^{p} w\right)}{U_{\beta}(|u|+p+n)}\right)+\frac{Q}{U_{\beta}(|u|+p+n)}\right)
$$

converges, for the Hausdorff distance, to the diagonal of the square $\left(0.0^{|u|-|v|} v, 0 . u\right)+Q / \beta^{|u|+p}$.

Example 31. As a first example, when $\beta=2$, we find back the construction in [10. As a second example, let us take $\beta=\varphi$ to be the golden ratio. Let $u=101$ and $v=10$ (resp., $u^{\prime}=100=v^{\prime}$ ). Then $p(u, v)=1$ (resp., $p\left(u^{\prime}, v^{\prime}\right)=0$ ); see Example 21. Those pairs of words satisfy $(\star)$. The first few terms of the sequence of sets (3) are respectively depicted in Figure 6 and Figure 7. Observe that when $n$ tends to infinity, the union of black squares in $\mathcal{U}_{n+4}^{\varphi}$ (resp., $\left.\mathcal{U}_{n+3}^{\varphi}\right)$ converges to the diagonal of $(0.0 v, 0 . u)+Q / \varphi^{4}$ (resp., $\left.\left(0 . v^{\prime}, 0 . u^{\prime}\right)+Q / \varphi^{3}\right)$. 


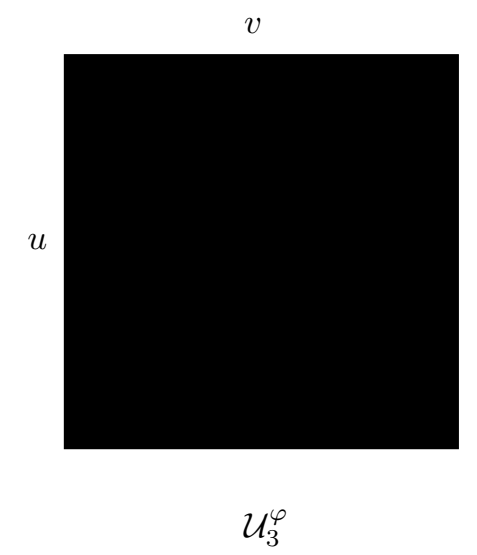

(a) A subset of $\mathcal{U}_{3}^{\varphi}$.

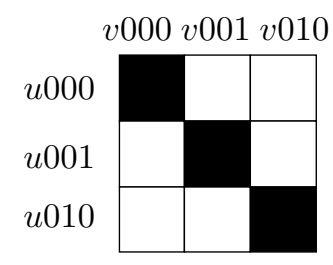

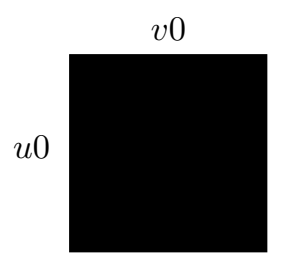

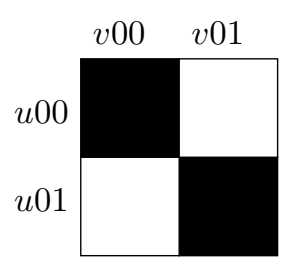

$\mathcal{U}_{4}^{\varphi}$

(b) The element $n=0$ of (3).

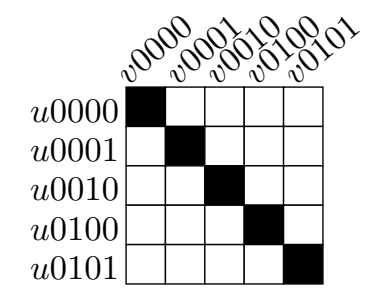

$\mathcal{U}_{6}^{\varphi}$

(d) The element $n=2$ of (3).
$\mathcal{U}_{7}^{\varphi}$

(e) The element $n=3$ of (3).

Figure 6: The first few terms of sequence of sets $(3)$ converging to the diagonal of the square $(0.0 v, 0 . u)+Q / \varphi^{4}$ for $u=101$ and $v=10$. 

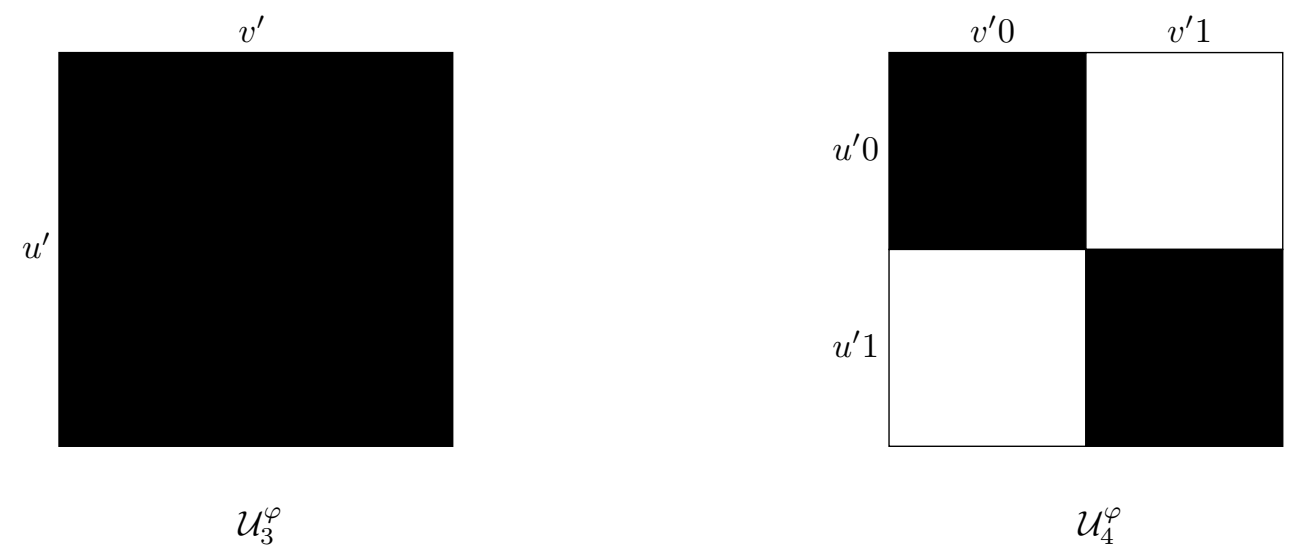

(a) The element $n=0$ of (3).

(b) The element $n=1$ of 3 .
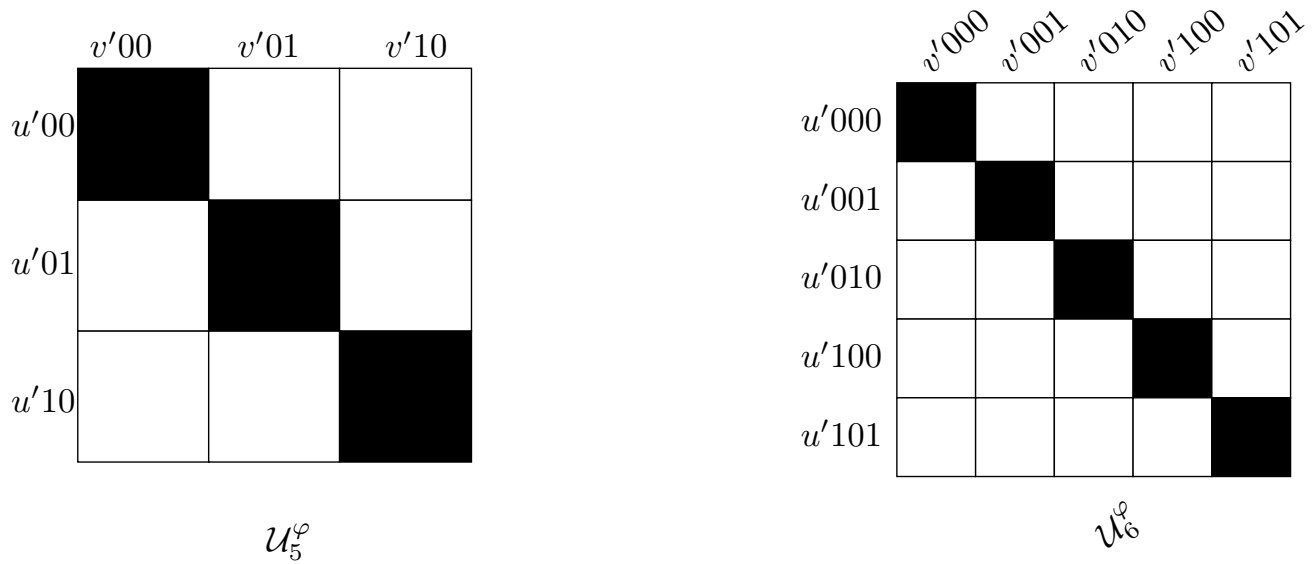

(c) The element $n=2$ of 3 .

(d) The element $n=3$ of 3 .

Figure 7: The first few terms of sequence of sets (3) converging to the diagonal of the square $\left(0 . v^{\prime}, 0 . u^{\prime}\right)+Q / \varphi^{3}$ for $u^{\prime}=100$ and $v^{\prime}=100$. 


\section{The sequence of compact sets $\left(\mathcal{A}_{n}^{\beta}\right)_{n \geq 0}$}

The observation made in Remark 30 leads to the definition of an initial set $\mathcal{A}_{0}^{\beta}$. The same technique is applied in [10. At first, let us define a segment associated with a pair of words.

Definition 32. Let $(u, v)$ in $L_{U_{\beta}} \times L_{U_{\beta}}$ such that $|u| \geq|v| \geq 0$. We define a closed segment $S_{u, v}$ of slope 1 and of length $\sqrt{2} \cdot \beta^{-|u|-p(u, v)}$ in $[0,1] \times[0,1]$. The endpoints of $S_{u, v}$ are given by $A_{u, v}:=\left(0.0^{|u|-|v|} v, 0 . u\right)$ and

$$
B_{u, v}:=A_{u, v}+\left(\beta^{-|u|-p(u, v)}, \beta^{-|u|-p(u, v)}\right)=\left(0.0^{|u|-|v|} v 0^{p(u, v)} d_{\beta}^{*}(1), 0 . u 0^{p(u, v)} d_{\beta}^{*}(1)\right) .
$$

Observe that, if $u=v=\varepsilon$, the associated segment of slope 1 has endpoints $(0,0)$ and $(1,1)$. Otherwise, the segment $S_{u, v}$ lies in $[0,1] \times[1 / \beta, 1]$.

Definition 33. Let us define the following compact set which is the closure of a countable union of segments

$$
\mathcal{A}_{0}^{\beta}:=\bigcup_{\substack{(u, v) \\ \text { satisfying }(\star)}} S_{u, v} .
$$

Notice that Definition 32 implies that $\mathcal{A}_{0}^{\beta} \subset[0,1] \times[0,1]$. More precisely, $\mathcal{A}_{0}^{\beta} \backslash S_{\varepsilon, \varepsilon} \subset[0,1] \times[1 / \beta, 1]$. Furthermore, observe that we take the closure of a union to ensure the compactness of the set.

Example 34. Let $\beta=\varphi$ be the golden ratio. In Figure 8, the segment $S_{u, v}$ is represented for all $(u, v)$ satisfying $(\star)$ and such that $0 \leq|v| \leq|u| \leq 10$.

In the following definition, we introduce another sequence of compact sets obtained by transforming the initial set $\mathcal{A}_{0}^{\beta}$ under iterations of two maps. This new sequence, which is shown to be a Cauchy sequence in Proposition 36, allows us to define properly the limit set $\mathcal{L}^{\beta}$.

Definition 35. We let $c$ denote the homothety of center $(0,0)$ and ratio $1 / \beta$ and we consider the map $h:(x, y) \mapsto(x, \beta y)$. We define a sequence of compact sets by setting, for all $n \geq 0$,

$$
\mathcal{A}_{n}^{\beta}:=\bigcup_{\substack{0 \leq i \leq n \\ 0 \leq j \leq i}} h^{j}\left(c^{i}\left(\mathcal{A}_{0}^{\beta}\right)\right) .
$$

In Figure 9, we apply $c$ and $h$ at most twice from $\mathcal{A}_{0}^{\beta} \backslash S_{\varepsilon, \varepsilon}$. Let $m, n$ with $m \leq n$. Using Figure 9 , observe that

$$
\mathcal{A}_{m}^{\beta} \cap\left(\left[1 / \beta^{m+1}, 1\right] \times[0,1]\right)=\mathcal{A}_{n}^{\beta} \cap\left(\left[1 / \beta^{m+1}, 1\right] \times[0,1]\right) .
$$

Proposition 36. The sequence $\left(\mathcal{A}_{n}^{\beta}\right)_{n \geq 0}$ is a Cauchy sequence.

Proof. Let $\epsilon>0$ and take $n>m$. We must show that $\mathcal{A}_{m}^{\beta} \subset\left[\mathcal{A}_{n}^{\beta}\right]_{\epsilon}$ and $\mathcal{A}_{n}^{\beta} \subset\left[\mathcal{A}_{m}^{\beta}\right]_{\epsilon}$. The first inclusion is easy. Indeed, since $\mathcal{A}_{m}^{\beta} \subset \mathcal{A}_{n}^{\beta}$, we directly have that $\left[\mathcal{A}_{n}^{\beta}\right]_{\epsilon}$ contains $\mathcal{A}_{m}^{\beta}$. Let us show the second inclusion. From (4), $\mathcal{A}_{m}^{\beta}$ and consequently $\left[\mathcal{A}_{m}^{\beta}\right]_{\epsilon}$ both contain $\mathcal{A}_{n}^{\beta} \cap\left(\left[1 / \beta^{m+1}, 1\right] \times[0,1]\right)$. Now we show that $\left[\mathcal{A}_{m}^{\beta}\right]_{\epsilon}$ contains $\left[0,1 / \beta^{m+1}\right) \times[0,1]$ if $m$ is sufficiently large, which ends the proof. By Definition $33 \mathcal{A}_{0}^{\beta}$ contains the segment $S_{\varepsilon, \varepsilon}$ of slope 1 with endpoints $(0,0)$ and $(1,1)$. Thus, by Definition $35, \mathcal{A}_{m}^{\beta}$ contains the segment $h^{m}\left(c^{m}\left(S_{\varepsilon, \varepsilon}\right)\right)$ of slope $\beta^{m}$ with endpoints $(0,0)$ and $\left(1 / \beta^{m}, 1\right)$. Let $(x, y) \in\left[0,1 / \beta^{m+1}\right) \times[0,1]$. Then $\left(y / \beta^{m}, y\right)$ belongs to $h^{m}\left(c^{m}\left(S_{\varepsilon, \varepsilon}\right)\right) \subset \mathcal{A}_{m}^{\beta}$. Consequently,

$$
d\left((x, y), \mathcal{A}_{m}^{\beta}\right) \leq d\left((x, y),\left(y / \beta^{m}, y\right)\right) \leq x+y / \beta^{m}<\epsilon
$$

if $m$ is sufficiently large. 


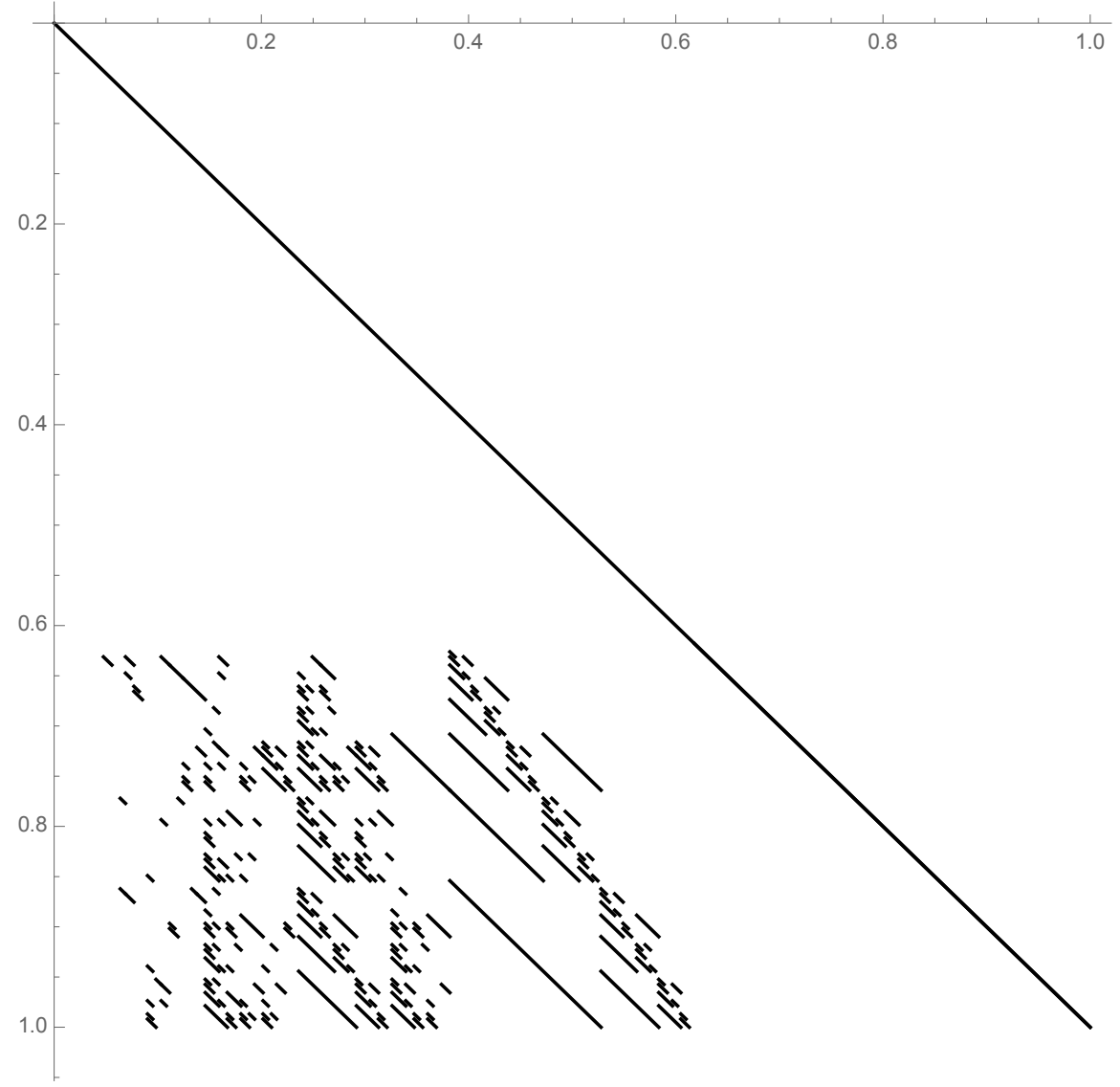

Figure 8: An approximation of $\mathcal{A}_{0}^{\varphi}$ computed with words of length $\leq 10$. 


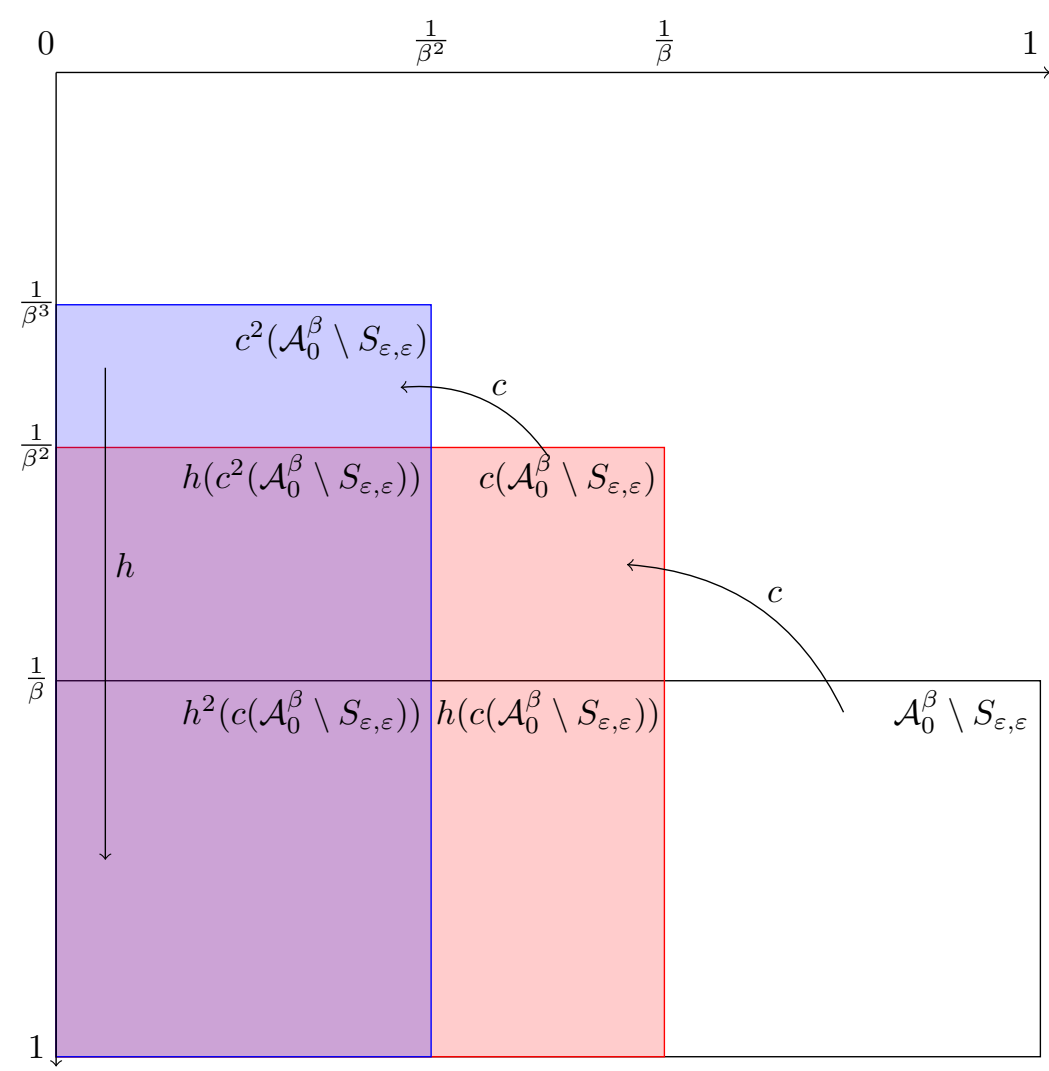

Figure 9: Two applications of $c$ and $h$ from $\mathcal{A}_{0}^{\beta} \backslash S_{\varepsilon, \varepsilon}$.

Definition 37. Since the sequence $\left(\mathcal{A}_{n}^{\beta}\right)_{n \geq 0}$ is a Cauchy sequence in the complete metric space $\left(\mathcal{H}\left(\mathbb{R}^{2}\right), d_{h}\right)$, its limit is a well-defined compact set denoted by $\mathcal{L}^{\beta}$.

Example 38. Let $\varphi$ be the golden ratio. We have represented in Figure 10 all the segments of $\mathcal{A}_{0}^{\varphi}$ for words of length at most 10 and we have applied the maps $h^{j}\left(c^{i}(\cdot)\right)$ to this set of segments for $0 \leq j \leq i \leq 4$. Thus we have an approximation of $\mathcal{A}_{4}^{\varphi}$.

\section{The limit of the sequence of compact sets $\left(\mathcal{U}_{n}^{\beta}\right)_{n \geq 0}$}

In this section, we show that the sequence $\left(\mathcal{U}_{n}^{\beta}\right)_{n \geq 0}$ of compact subsets of $[0,1] \times[0,1]$ also converges to $\mathcal{L}^{\beta}$. The proofs of Lemma 39, Lemma 44 are essentially the same as the ones from [10] (10, Lemma 27, Lemma 28, Theorem 29]). However we recall them so that the paper is self-contained. The first part is to show that, when $\epsilon$ is a positive real number, then $\mathcal{U}_{n}^{\beta} \subset\left[\mathcal{L}^{\beta}\right]_{\epsilon}$ for all sufficiently large $n$.

Lemma 39. Let $\epsilon>0$. For all sufficiently large $n \in \mathbb{N}$, we have

$$
\mathcal{U}_{n}^{\beta} \subset\left[\mathcal{L}^{\beta}\right]_{\epsilon} .
$$

Proof. Let $\epsilon>0$. Take $n \in \mathbb{N}$ and let $(x, y) \in \mathcal{U}_{n}^{\beta}$. From Remark 18 , there exists $(u, v) \in L_{U_{\beta}} \times L_{U_{\beta}}$ such that $\left(\begin{array}{l}u \\ v\end{array}\right) \equiv 1 \bmod 2,0 \leq|v| \leq|u| \leq n$ and the point $(x, y)$ belongs to the square region

$$
\left(\left(\operatorname{val}_{U_{\beta}}(v), \operatorname{val}_{U_{\beta}}(u)\right)+Q\right) / U_{\beta}(n) \subset \mathcal{U}_{n}^{\beta} .
$$

Let us set

$$
A:=\left(\frac{\operatorname{val}_{U_{\beta}}(v)}{U_{\beta}(n)}, \frac{\operatorname{val}_{U_{\beta}}(u)}{U_{\beta}(n)}\right)
$$

to be the upper-left corner of the square region (5) in $\mathcal{U}_{n}^{\beta}$. 


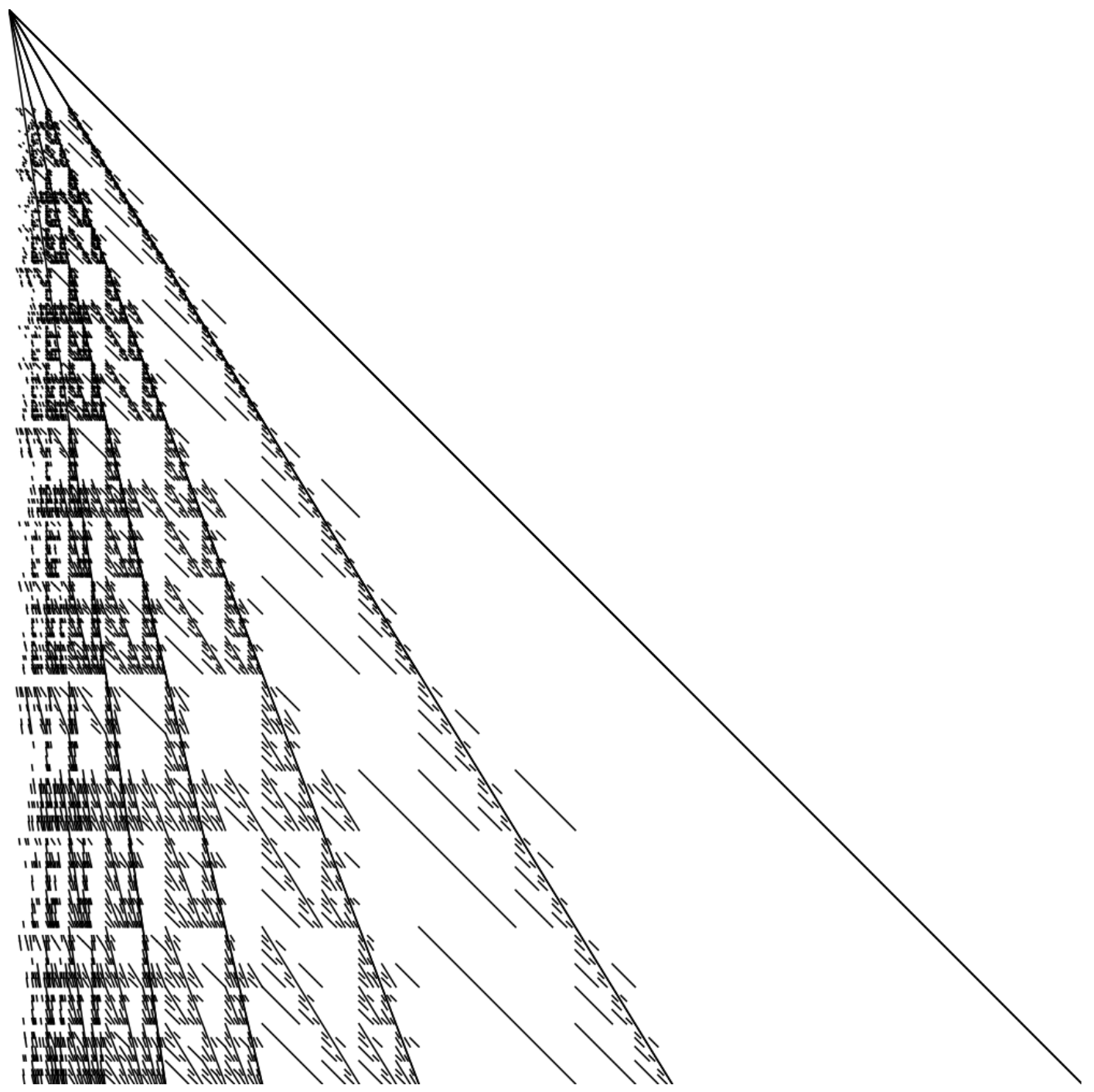

Figure 10: An approximation of the limit set $\mathcal{L}^{\varphi}$.

Assume first that $(u, v)$ satisfies $(\star)$. The segment $S_{u, v}$ of length $\sqrt{2} \cdot \beta^{-|u|-p(u, v)}$ having $A_{u, v}=$ $\left(0.0^{|u|-|v|} v, 0 . u\right)$ as endpoint belongs to $\mathcal{A}_{0}^{\beta}$. Now apply $n-|u|$ times the homothety $c$ to this segment. So the segment $c^{n-|u|}\left(S_{u, v}\right)$ of length $\sqrt{2} \cdot \beta^{-n-p(u, v)}$ of endpoint $B_{1}:=\left(0.0^{n-|v|} v, 0.0^{n-|u|} u\right)$ belongs to $\mathcal{A}_{n-|u|}^{\beta}$ and thus to $\mathcal{L}^{\beta}$. Using (2) (the reasoning is similar to the one developed in the proof of Lemma 29), there exists $N_{1} \in \mathbb{N}$ such that, for all $n \geq N_{1}, d\left(A, B_{1}\right)<\epsilon / 2$. Hence, for all $n \geq N_{1}$ such that $\sqrt{2} / U_{\beta}(n)<\epsilon / 2$, we have

$$
d\left((x, y), \mathcal{L}^{\beta}\right) \leq d\left((x, y), B_{1}\right) \leq d((x, y), A)+d\left(A, B_{1}\right) \leq \sqrt{2} / U_{\beta}(n)+d\left(A, B_{1}\right)<\epsilon .
$$

Now assume that $(u, v)$ does not satisfy $(\star)$. Since $\left(\begin{array}{l}u \\ v\end{array}\right) \equiv 1 \bmod 2$, then either $u$ and $v$ are non-empty words, or $u$ is non-empty and $v=\varepsilon$. Suppose that $u$ and $v$ are non-empty. By assumption, we have an odd number $r$ of occurrences of $v$ in $u$. For each occurrence of $v$ in $u$, we count the total number of zeroes after it. We thus define a sequence of non-negative integer indices

$$
|u| \geq i_{1} \geq i_{2} \geq \cdots \geq i_{r} \geq 0
$$


corresponding to the number of zeroes following the first, the second, ..., the $r$ th occurrence of $v$ in $u$. Now let $k$ be a non-negative integer such that $k>\left\lceil\log _{2}|u|\right\rceil$ and $2^{k}>p(u, v)$. By definition of $p(u, v)$, the words $u 0^{2^{k}} 1$ and $v 0^{2^{k}} 1$ belong to $L_{U_{\beta}}$. We get

$$
\left(\begin{array}{l}
u 0^{2^{k}} 1 \\
v 0^{2^{k}} 1
\end{array}\right)=\sum_{\ell=1}^{r}\left(\begin{array}{c}
2^{k}+i_{\ell} \\
2^{k}
\end{array}\right)
$$

Indeed, for each $\ell \in\{1, \ldots, r\}$, consider the $\ell$ th occurrence of $v$ in $u$ : we have the factorization $u=p w$ where the last letter of $p$ is the last letter of the $\ell$ th occurrence of $v$ and $|w|_{0}=i_{\ell}$. With this particular occurrence of $v$, we obtain occurrences of $v 0^{2^{k}} 1$ in $u 0^{2^{k}} 1$ by choosing $2^{k}$ zeroes among the $2^{k}+i_{\ell}$ zeroes available in $w 0^{2^{k}} 1$. Moreover, with the long block of $2^{k}$ zeroes, it is not possible to have any other occurrence of $v 0^{2^{k}} 1$ than those obtained from occurrences of $v$ in $u$.

Then, for each $\ell \in\{1, \ldots, r\}$, we have

$$
\left(\begin{array}{c}
2^{k}+i_{\ell} \\
2^{k}
\end{array}\right) \equiv 1 \bmod 2
$$

from Theorem 2. Since $r$ is odd, we get

$$
\left(\begin{array}{l}
u 0^{2^{k}} 1 \\
v 0^{2^{k}} 1
\end{array}\right) \equiv 1 \bmod 2
$$

Now, for all $k \in \mathbb{N}$ such that $k>\left\lceil\log _{2}|u|\right\rceil$ and $2^{k}>p(u, v)$, it is easy to check that the pair of words $\left(u 0^{2^{k}} 1, v 0^{2^{k}} 1\right)$ satisfies $(\star)$. For the sake of simplicity, define $u_{k}:=u 0^{2^{k}} 1, v_{k}:=v 0^{2^{k}} 1$ and $p_{k}:=$ $p\left(u_{k}, v_{k}\right)$. As in the first part of the proof, the segment $S_{u_{k}, v_{k}}$ of length $\sqrt{2} \cdot \beta^{-|u|-2^{k}-1-p_{k}}$ having $A_{u_{k}, v_{k}}=$ $\left(0.0^{|u|-|v|} v 0^{2^{k}} 1,0 . u 0^{2^{k}} 1\right)$ as endpoint belongs to $\mathcal{A}_{0}^{\beta}$. Now apply $n-|u|$ times the homothety $c$ to this segment. So the segment $c^{n-|u|}\left(S_{u_{k}, v_{k}}\right)$ of length $\sqrt{2} \cdot \beta^{-n-2^{k}-1-p_{k}}$ of endpoint $B_{2}:=\left(0.0^{n-|v|} v 0^{2^{k}} 1,0.0^{n-|u|} u 0^{2^{k}} 1\right)$ belongs to $\mathcal{A}_{n-|u|}^{\beta}$ and thus to $\mathcal{L}^{\beta}$. Using again 22 and a reasoning similar to the one from the proof of Lemma 29, there exists $N_{2} \in \mathbb{N}$ such that, for all $n \geq N_{2}, d\left(A, B_{2}\right)<\epsilon / 2$. Hence, for all $n \geq N_{2}$ such that $\sqrt{2} / U_{\beta}(n)<\epsilon / 2$, we have

$$
d\left((x, y), \mathcal{L}^{\beta}\right) \leq d\left((x, y), B_{2}\right) \leq d((x, y), A)+d\left(A, B_{2}\right) \leq \sqrt{2} / U_{\beta}(n)+d\left(A, B_{2}\right)<\epsilon .
$$

Assume now that $u$ is non-empty and $v=\varepsilon$. In this case, the point $A$ is on the vertical line of equation $x=0$. By Definition 33, $\mathcal{A}_{0}^{\beta}$ contains the segment $S_{\varepsilon, \varepsilon}$ of slope 1 with endpoints $(0,0)$ and $(1,1)$. Thus, by Definition 35, $\mathcal{A}_{n}^{\beta}$ contains the segment $h^{n}\left(c^{n}\left(S_{\varepsilon, \varepsilon}\right)\right)$ of slope $\beta^{n}$ with endpoints $(0,0)$ and $\left(1 / \beta^{n}, 1\right)$. This segment also lies in $\mathcal{L}^{\beta}$. There exists $N_{3} \in \mathbb{N}$ such that, for all $n \geq N_{3}, d\left(A, h^{n}\left(c^{n}\left(S_{\varepsilon, \varepsilon}\right)\right)\right) \leq 1 / \beta^{n}<\epsilon / 2$. Consequently, for all $n \geq N_{3}$ such that $\sqrt{2} / U_{\beta}(n)<\epsilon / 2$, we have

$$
\begin{aligned}
d\left((x, y), \mathcal{L}^{\beta}\right) & \leq d\left((x, y), h^{n}\left(c^{n}\left(S_{\varepsilon, \varepsilon}\right)\right)\right) \leq d((x, y), A)+d\left(A, h^{n}\left(c^{n}\left(S_{\varepsilon, \varepsilon}\right)\right)\right) \\
& \leq \sqrt{2} / U_{\beta}(n)+d\left(A, h^{n}\left(c^{n}\left(S_{\varepsilon, \varepsilon}\right)\right)\right)<\epsilon .
\end{aligned}
$$

In each of the three cases, we conclude that $(x, y) \in\left[\mathcal{L}^{\beta}\right]_{\epsilon}$, which proves that $\mathcal{U}_{n}^{\beta} \subset\left[\mathcal{L}^{\beta}\right]_{\epsilon}$ for all sufficiently large $n$.

If $\epsilon>0$, it remains to show that $\mathcal{L}^{\beta} \subset\left[U_{n}\right]_{\epsilon}$ for all sufficiently large $n \in \mathbb{N}$. To that aim, we need to bound the number of consecutive words, in the genealogical order, that end with 0 in $L_{U_{\beta}}$.

Definition 40. We let $C_{\beta} \in \mathbb{N}$ denote the maximal number of consecutive 0 in $d_{\beta}^{*}(1)$, i.e.,

$$
C_{\beta}:=\max \left\{n \in \mathbb{N} \mid 0^{n} \text { is a factor of } d_{\beta}^{*}(1)\right\} .
$$




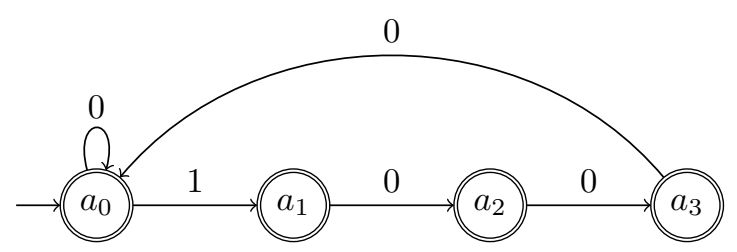

Figure 11: The automaton $\mathcal{A}_{\beta}$ for the dominant root $\beta$ of the polynomial $P(X)=X^{4}-X^{3}-1$.

In the next proposition, we show that the maximal number of consecutive words ending with 0 in $L_{U_{\beta}}$ is $C_{\beta}+1$.

Proposition 41. If we order the words in $L_{U_{\beta}}$ by the genealogical order, the maximal number of consecutive words ending with 0 in $L_{U_{\beta}}$, i.e., the maximal number of consecutive normal $U_{\beta}$-representations ending with 0 , is $C_{\beta}+1$.

Proof. Let $n \in \mathbb{N}$ be such that $\operatorname{rep}_{U_{\beta}}(n)$ ends with 0 . We can suppose that $\operatorname{rep}_{U_{\beta}}(n-1)$ does not end with 0 , otherwise we translate $n$. If $\left|\operatorname{rep}_{U_{\beta}}(n+1)\right|=\left|\operatorname{rep}_{U_{\beta}}(n)\right|$, then $\operatorname{rep}_{U_{\beta}}(n+1)$ does not end with 0 because $U_{\beta}(m) \geq 2$ for all $m \geq 1$. Indeed, if a single digit (not the least significant one) is changed, then the value is increased by at least 2 . Let $C \geq 1$ be such that, for all $k \in\{0, \ldots, C\},\left|\operatorname{rep}_{U_{\beta}}(n+k)\right|=\left|\operatorname{rep}_{U_{\beta}}(n)\right|+k$ and $\left|\operatorname{rep}_{U_{\beta}}(n+C+1)\right|=\left|\operatorname{rep}_{U_{\beta}}(n+C)\right|$. The normal- $U$ representation preserves the order, i.e., for all integers $m_{1}$ and $m_{2}, m_{1} \leq m_{2}$ if and only if $\operatorname{rep}_{U_{\beta}}\left(m_{1}\right) \leq \operatorname{rep}_{U_{\beta}}\left(m_{2}\right)$ (see, for instance, [3). Thus, the words $\operatorname{rep}_{U_{\beta}}(n), \ldots, \operatorname{rep}_{U_{\beta}}(n+C-1)$ are prefixes of $d_{\beta}^{*}(1)$, respectively of length $\left|\operatorname{rep}_{U_{\beta}}(n)\right|,\left|\operatorname{rep}_{U_{\beta}}(n)\right|+$ $1, \ldots,\left|\operatorname{rep}_{U_{\beta}}(n)\right|+C-1$ (the prefixes of $d_{\beta}^{*}(1)$ are the maximal words of different length in $L_{U_{\beta}}$ ). By Definition 40, we deduce that $C \leq C_{\beta}$. Consequently, there are at most $C_{\beta}+1$ consecutive words ending with 0 in $L_{U_{\beta}}$.

Let us illustrate the previous proposition.

Example 42. Let $\varphi$ be the golden ratio. Then $C_{\varphi}=1$ since $d_{\varphi}^{*}(1)=(10)^{\omega}$. The first few words of $L_{U_{\varphi}}$ are $\varepsilon, 1,10,100,101,1000,1001,1010,10000,10001, \ldots$ The maximal number of consecutive words ending with 0 in $L_{U_{\varphi}}$ is $C_{\varphi}+1=2$.

Example 43. Let $\beta$ be the dominant root of the polynomial $P(X)=X^{4}-X^{3}-1$. Then $\beta \approx 1.38028$ is a Parry number with $d_{\beta}(1)=1001$ and $d_{\beta}^{*}(1)=(1000)^{\omega}$. The automaton $\mathcal{A}_{\beta}$ is depicted in Figure 11. In this example, $C_{\beta}=3$. The first few words of $L_{U_{\beta}}$ are $\varepsilon, 1,10,100,1000,10000,10001, \ldots$. The maximal number of consecutive words ending with 0 in $L_{U_{\beta}}$ is $C_{\beta}+1=4$.

Lemma 44. Let $\epsilon>0$. For all $(x, y) \in \mathcal{L}^{\beta}, d\left((x, y), \mathcal{U}_{n}^{\beta}\right)<\epsilon$ for all sufficiently large $n$.

Proof. Let $\epsilon>0$ and let $(x, y) \in \mathcal{L}^{\beta}$. Since $\left(\mathcal{A}_{n}^{\beta}\right)_{n \geq 0}$ converges to $\mathcal{L}^{\beta}$, there exists $N_{1}$ and $\left(x^{\prime}, y^{\prime}\right) \in \mathcal{A}_{N_{1}}^{\beta}$ such that,

$$
d\left((x, y),\left(x^{\prime}, y^{\prime}\right)\right)<\epsilon / 4 .
$$

By definition of $\mathcal{A}_{N_{1}}^{\beta}$, there exist $i, j$ such that $0 \leq j \leq i \leq N_{1}$ and $\left(x_{0}^{\prime}, y_{0}^{\prime}\right) \in \mathcal{A}_{0}^{\beta}$ such that

$$
h^{j}\left(c^{i}\left(\left(x_{0}^{\prime}, y_{0}^{\prime}\right)\right)\right)=\left(x^{\prime}, y^{\prime}\right) .
$$

By definition of $\mathcal{A}_{0}^{\beta}$, there exists a pair $(u, v) \in L_{U_{\beta}} \times L_{U_{\beta}}$ satisfying $(\star)$ and $\left(x_{0}^{\prime \prime}, y_{0}^{\prime \prime}\right) \in S_{u, v}$ such that

$$
d\left(\left(x_{0}^{\prime}, y_{0}^{\prime}\right),\left(x_{0}^{\prime \prime}, y_{0}^{\prime \prime}\right)\right)<\epsilon / 4 \text {. }
$$

Notice that, since $j \leq i$,

$$
\begin{aligned}
d\left(\left(x^{\prime}, y^{\prime}\right), h^{j}\left(c^{i}\left(\left(x_{0}^{\prime \prime}, y_{0}^{\prime \prime}\right)\right)\right)\right) & =d\left(h^{j}\left(c^{i}\left(\left(x_{0}^{\prime}, y_{0}^{\prime}\right)\right)\right), h^{j}\left(c^{i}\left(\left(x_{0}^{\prime \prime}, y_{0}^{\prime \prime}\right)\right)\right)\right) \\
& \leq d\left(\left(x_{0}^{\prime}, y_{0}^{\prime}\right),\left(x_{0}^{\prime \prime}, y_{0}^{\prime \prime}\right)\right)<\epsilon / 4 .
\end{aligned}
$$


Consequently, we get that

$$
d\left((x, y), h^{j}\left(c^{i}\left(\left(x_{0}^{\prime \prime}, y_{0}^{\prime \prime}\right)\right)\right)\right)<\epsilon / 2 .
$$

In the second part of the proof, we will show that $d\left(h^{j}\left(c^{i}\left(\left(x_{0}^{\prime \prime}, y_{0}^{\prime \prime}\right)\right)\right), \mathcal{U}_{n}^{\beta}\right)<\epsilon / 2$ for all sufficiently large $n$. We will make use of the constants $i, j$, the words $u, v$ given above and the integer $p:=p(u, v)$.

Set

$$
L_{u, v}:=\left\{\begin{array}{cl}
L_{U_{\beta}}, & \text { if } u=v=\varepsilon ; \\
0^{*} L_{U_{\beta}}, & \text { otherwise. }
\end{array}\right.
$$

Since $(u, v) \in L_{U_{\beta}} \times L_{U_{\beta}}$ satisfies $(\star)$, the pair of words $\left(u 0^{p} w, v 0^{p} w\right)$ has an odd binomial coefficient, for all words $w \in L_{u, v}$, using Lemma 25 and Corollary 28. In particular, this is the case when $w \in L_{u, v}$ is of length $n$. We can choose $n$ sufficiently large such that $U_{\beta}(n) \geq C_{\beta}+3$ using Proposition 41. In this case, there exist at least two words $w \in L_{u, v}$ with $|w|=n$ and not ending with 0 . Furthermore, as soon as $w$ does not end with 0 , Lemma 1 shows that

$$
\left(\begin{array}{c}
u 0^{p} w 0^{k} \\
v 0^{p} w
\end{array}\right)=\left(\begin{array}{c}
u 0^{p} w \\
v 0^{p} w
\end{array}\right) \equiv 1 \bmod 2
$$

for all $k \geq 0$. By definition of the sequence $U_{\beta}$, we also have

$$
\#\left\{z \in 0^{*} L_{U_{\beta}} \mid u 0^{p} w z \in L_{U_{\beta}} \text { and }|z|=k\right\} \leq U_{\beta}(k) .
$$

Thus, for all $j \leq i$, we conclude that at least one of the $U_{\beta}(j)$ binomial coefficients of the form $\left(\begin{array}{c}u 0^{p} w z \\ v 0^{p} w\end{array}\right)$ with $w$ not ending with 0 and $|z|=j$ is odd (indeed, choose $z=0^{j}$ for instance). Otherwise stated, at least one of the square regions

$$
\left(\frac{\operatorname{val}_{U_{\beta}}\left(v 0^{p} w\right)}{U_{\beta}(n+i+|u|+p)}, \frac{\operatorname{val}_{U_{\beta}}\left(u 0^{p} w z\right)}{U_{\beta}(n+i+|u|+p)}\right)+\frac{Q}{U_{\beta}(n+i+|u|+p)}, \text { with }|z|=j,
$$

is a subset of $\mathcal{U}_{n+i+|u|+p}^{\beta}$, since $\left|v 0^{p} w\right|,\left|u 0^{p} w z\right| \leq n+i+|u|+p$. This can be visualized in Figure 12 .

Now observe that, for any word $w \in L_{u, v}$, each square region of the form $(6)$ is intersected by $h^{j}\left(c^{\imath}\left(S_{u, v}\right)\right)$. Indeed, the latter segment has $A:=\left(0.0^{i+|u|-|v|} v, 0.0^{i-j} u\right)$ and $B:=\left(0.0^{i+|u|-|v|} v 0^{p} d_{\beta}^{*}(1), 0.0^{i-j} u 0^{p} d_{\beta}^{*}(1)\right)$ as endpoints and slope $\beta^{j}$. Using (2), if $n$ is sufficiently large, the points

$$
\left(\frac{\operatorname{val}_{U_{\beta}}\left(v 0^{p} 0^{n}\right)}{U_{\beta}(n+i+|u|+p)}, \frac{\operatorname{val}_{U_{\beta}}\left(u 0^{p} 0^{n+j}\right)}{U_{\beta}(n+i+|u|+p)}\right)\left(\operatorname{resp} .,\left(\frac{\operatorname{val}_{U_{\beta}}\left(v 0^{p} d_{n}\right)}{U_{\beta}(n+i+|u|+p)}, \frac{\operatorname{val}_{U_{\beta}}\left(u 0^{p} d_{n+j}\right)}{U_{\beta}(n+i+|u|+p)}\right)\right)
$$

and $A$ (resp., $B$ ) are close for all $j \leq i$, where $d_{n}$ denotes the prefix of length $n$ of $d_{\beta}^{*}(1)$ for all $n \geq 0$. When $u$ and $v$ are non-empty, this can be seen in Figure 13 where each rectangular gray region contains at least one square region from $\mathcal{U}_{n+i+|u|+p}^{\beta}$ (to draw this picture, we take the particular case of the golden ratio $\varphi$ and $i=2)$. When $u=v=\varepsilon$, Figure 13 is modified in the following way: simply replace each word of the forms $u 0^{\ell}, v 0^{\ell}$ by $\varepsilon$.

Consequently, every point of $h^{j}\left(c^{i}\left(S_{u, v}\right)\right)$ is at distance at most

$$
\frac{2 \cdot\left(C_{\beta}+2\right) \cdot U_{\beta}(j)}{U_{\beta}(n+i+|u|+p)}
$$

from a point in $\mathcal{U}_{n+i+|u|+p}^{\beta}$ when $n$ is sufficiently large. Indeed, either the point falls into a gray region from Figure 13, or not. In the first case, the point is at distance at most $U_{\beta}(j) / U_{\beta}(n+i+|u|+p)$ from a square region in $\mathcal{U}_{n+i+|u|+p}^{\beta}$; see Figure 12 . Observe that this square region is of the form (6) where $w$ does not end with 0 . Otherwise, the point falls into a (white) square region of the form

$$
\left(\frac{\operatorname{val}_{U_{\beta}}\left(v 0^{p} w\right)}{U_{\beta}(n+i+|u|+p)}, \frac{\operatorname{val}_{U_{\beta}}\left(u 0^{p} w^{\prime} z\right)}{U_{\beta}(n+i+|u|+p)}\right)+\frac{Q}{U_{\beta}(n+i+|u|+p)}, \text { with }|w|=\left|w^{\prime}\right|=n,|z|=j .
$$




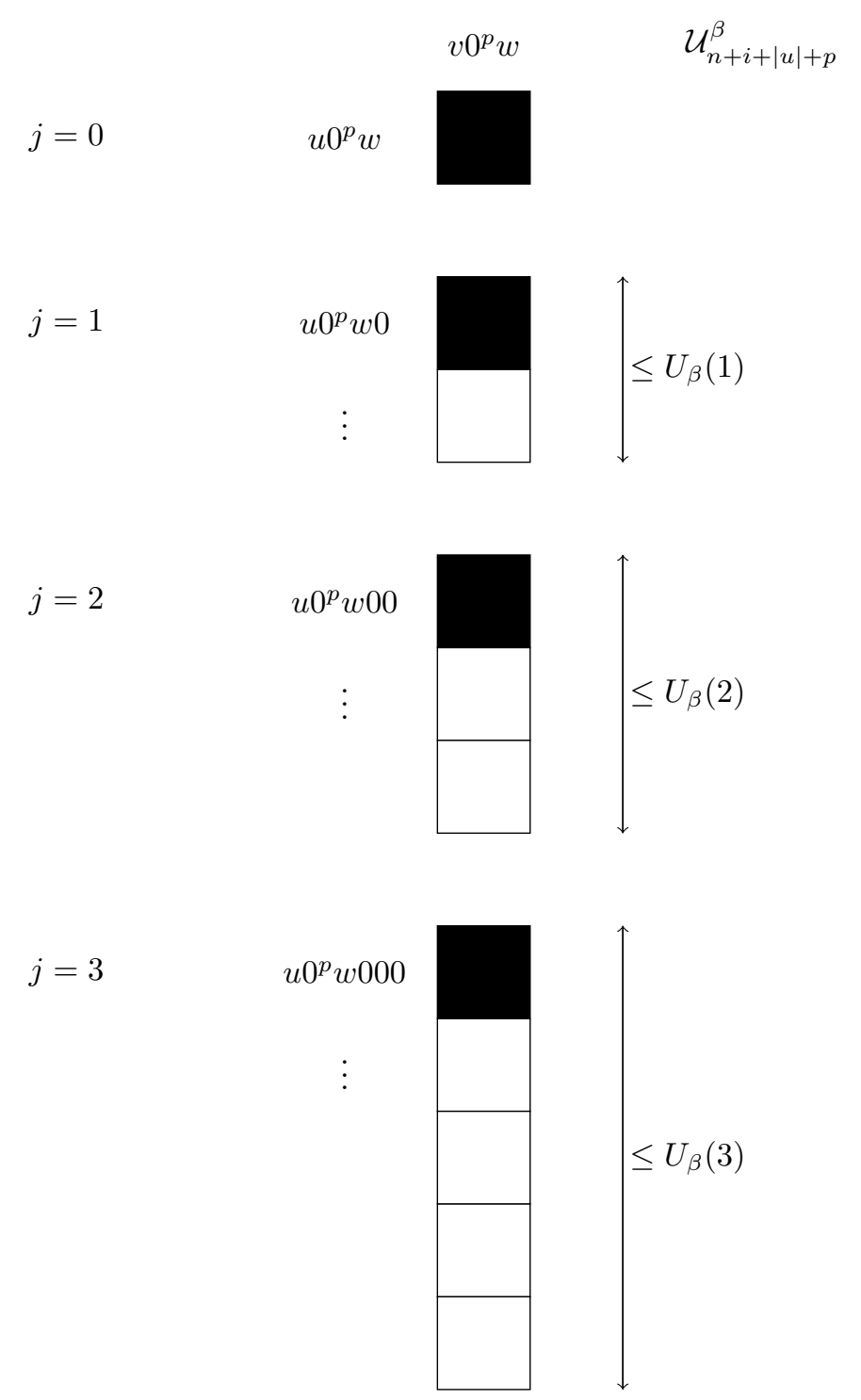

Figure 12: If $w$ does not end with 0 and is such that $|w|=n$, then $\left(\begin{array}{c}u 0^{p} w 0^{j} \\ v 0^{p} w\end{array}\right)$ being odd creates a square region in $\mathcal{U}_{n+i+|u|+p}^{\beta}$. 


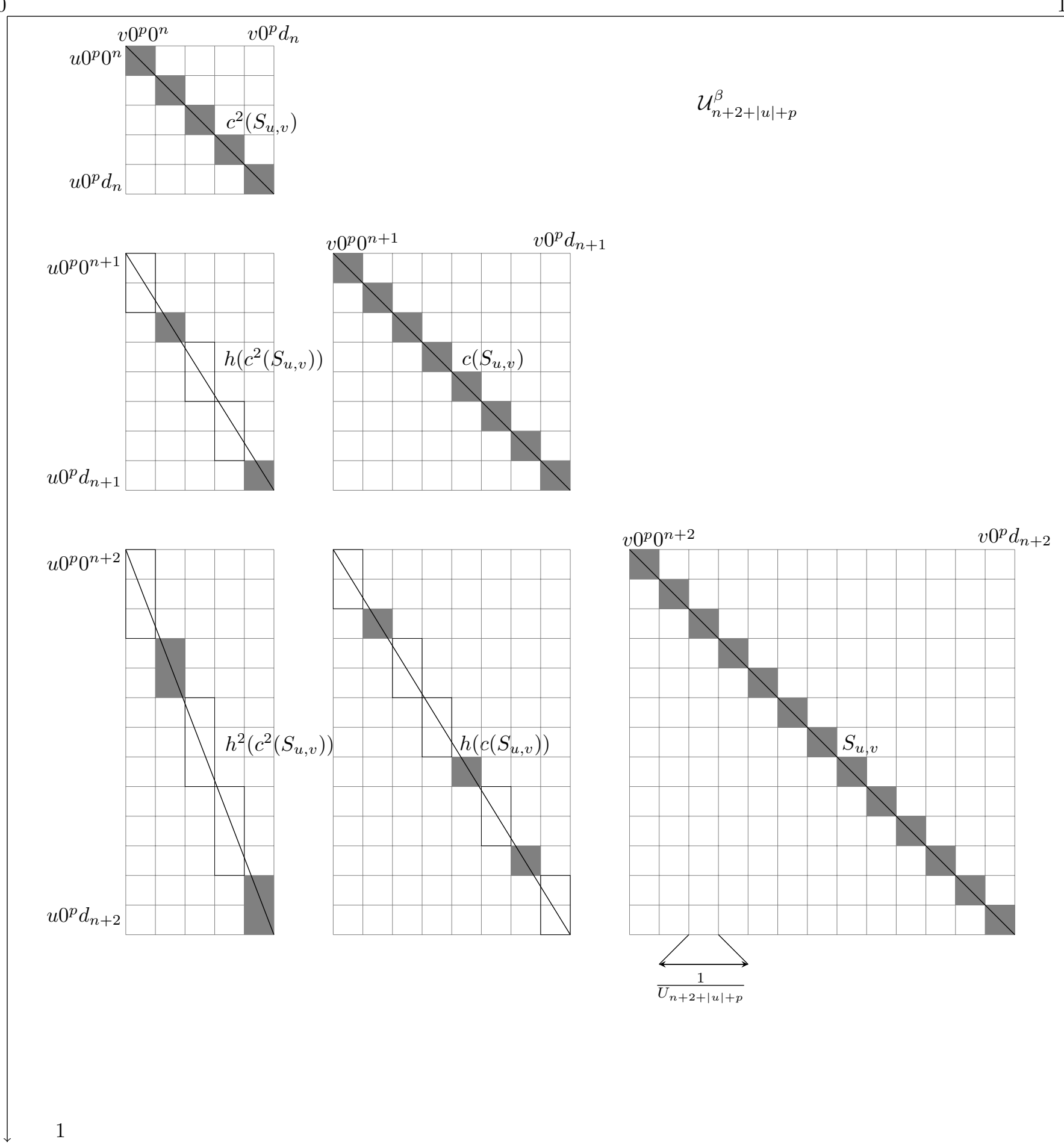

Figure 13: The situation occurring in the proof of Lemma 44 , where we choose $\beta$ to be the golden ratio. 
Since $n$ is large enough, there exists a word $w^{\prime \prime}$ not ending with 0 with $\left|w^{\prime \prime}\right|=n$, which is within a distance of $2 \cdot\left(C_{\beta}+2\right)$ of $w$ and $w^{\prime}$. Then, applying the argument from the previous case proves the statement.

In particular, the result holds for the point $h^{j}\left(c^{i}\left(\left(x_{0}^{\prime \prime}, y_{0}^{\prime \prime}\right)\right)\right)$ belonging to $h^{j}\left(c^{i}\left(S_{u, v}\right)\right)$. Hence, for all sufficiently large $n$,

$$
d\left(h^{j}\left(c^{i}\left(\left(x_{0}^{\prime \prime}, y_{0}^{\prime \prime}\right)\right)\right), \mathcal{U}_{n}^{\beta}\right)<\epsilon / 2 .
$$

The conclusion follows.

Corollary 45. Let $(u, v) \in L_{U_{\beta}} \times L_{U_{\beta}}$ satisfying $(\star)$ and let $0 \leq j \leq i$. For every point $(f, g)$ of the segment $h^{j}\left(c^{i}\left(S_{u, v}\right)\right)$, there exists a sequence $\left(\left(f_{n}, g_{n}\right)\right)_{n \geq 0}$ converging to $(f, g)$ and such that $\left(f_{n}, g_{n}\right) \in \mathcal{U}_{n}^{\beta}$ for all $n$.

Proof. Let $(f, g)$ be a point of the segment $h^{j}\left(c^{i}\left(S_{u, v}\right)\right)$. From the proof of Lemma 44 , we have

$$
d\left((f, g), \mathcal{U}_{m}^{\beta}\right) \leq \frac{2 \cdot\left(C_{\beta}+2\right) \cdot U_{\beta}(j)}{U_{\beta}(m)}
$$

for all sufficiently large $m$. Consequently, there exists a sequence $\left(\left(f_{n}, g_{n}\right)\right)_{n \geq 0}$ converging to $(f, g)$ and such that $\left(f_{n}, g_{n}\right) \in \mathcal{U}_{n}^{\beta}$ for all $n$.

We are now ready to prove the main result of this paper.

Theorem 46. The sequence $\left(\mathcal{U}_{n}^{\beta}\right)_{n \geq 0}$ converges to $\mathcal{L}^{\beta}$.

Proof. Let $\epsilon>0$. From Lemma 39 , it suffices to show that $\mathcal{L}^{\beta} \subset\left[\mathcal{U}_{n}^{\beta}\right]_{\epsilon}$ for all sufficiently large $n \in \mathbb{N}$. For all $(x, y) \in \mathcal{L}^{\beta}$, using Corollary 45 , there exists a (Cauchy) sequence $\left(\left(f_{i}(x, y), g_{i}(x, y)\right)_{i \geq 0}\right.$ such that $\left(f_{i}(x, y), g_{i}(x, y)\right) \in \mathcal{U}_{i}^{\beta}$ for all $i$, and there exists $N_{(x, y)}$ such that, for all $i, j \geq N_{(x, y)}$,

$$
d\left(\left(f_{i}(x, y), g_{i}(x, y)\right),\left(f_{j}(x, y), g_{j}(x, y)\right)\right)<\epsilon / 2
$$

and

$$
d\left(\left(f_{i}(x, y), g_{i}(x, y)\right),(x, y)\right)<\epsilon / 2
$$

We trivially have

$$
\mathcal{L}^{\beta} \subset \bigcup_{(x, y) \in \mathcal{L}^{\beta}} B\left(\left(f_{N_{(x, y)}}(x, y), g_{N_{(x, y)}}(x, y)\right), \epsilon / 2\right) .
$$

Since $\mathcal{L}^{\beta}$ is compact, we can extract a finite covering: there exist $\left(x_{1}, y_{1}\right), \ldots,\left(x_{k}, y_{k}\right)$ in $\mathcal{L}^{\beta}$ such that

$$
\mathcal{L}^{\beta} \subset \bigcup_{j=1}^{k} B\left(\left(f_{N_{\left(x_{j}, y_{j}\right)}}\left(x_{j}, y_{j}\right), g_{N_{\left(x_{j}, y_{j}\right)}}\left(x_{j}, y_{j}\right)\right), \epsilon / 2\right) .
$$

Let $N=\max _{j=1, \ldots, k} N_{\left(x_{j}, y_{j}\right)}$. From (7), we deduce that, for all $j \in\{1, \ldots, k\}$ and all $n \geq N$,

$$
B\left(\left(f_{N_{\left(x_{j}, y_{j}\right)}}\left(x_{j}, y_{j}\right), g_{N_{\left(x_{j}, y_{j}\right)}}\left(x_{j}, y_{j}\right)\right), \epsilon / 2\right) \subset B\left(\left(f_{n}\left(x_{j}, y_{j}\right), g_{n}\left(x_{j}, y_{j}\right), \epsilon\right)\right.
$$

and therefore

$$
\mathcal{L}^{\beta} \subset \bigcup_{j=1}^{k} B\left(\left(f_{n}\left(x_{j}, y_{j}\right), g_{n}\left(x_{j}, y_{j}\right)\right), \epsilon\right) \subset\left[\mathcal{U}_{n}^{\beta}\right]_{\epsilon} .
$$


Remark 47. As in [10], the results mentioned above can be extended to any prime number. Let $q$ be a prime number and $r$ be a positive residue in $\{1, \ldots, q-1\}$. We can extend Definition 16 to

$$
\mathcal{U}_{n, r}^{\beta}:=\frac{1}{U_{\beta}(n)} \bigcup\left\{\left(\operatorname{val}_{U_{\beta}}(v), \operatorname{val}_{U_{\beta}}(u)\right)+Q \mid u, v \in L_{U_{\beta}},\left(\begin{array}{l}
u \\
v
\end{array}\right) \equiv r \bmod q\right\} \subset[0,1] \times[0,1] .
$$

Since we make use of Lucas' theorem, we limit ourselves to congruences modulo a prime number. We just sketch the main differences with the case $q=2$.

See, for instance, Figure 15 for the case $\beta=\varphi, q=3$ and $r=2$.

The $(\star)$ condition from Definition 23 becomes $(\star)_{r}$. We say that $(u, v) \in L_{U_{\beta}} \times L_{U_{\beta}}$ satisfies the $(\star)_{r}$ condition if either $u=v=\varepsilon$ and $\left(\begin{array}{l}u \\ v\end{array}\right) \equiv r \bmod q$, or $|u| \geq|v|>0$ and

$$
\left(\begin{array}{l}
u 0^{p(u, v)} \\
v 0^{p(u, v)}
\end{array}\right) \equiv r \bmod q \quad \text { and } \quad\left(\begin{array}{c}
u 0^{p(u, v)} \\
v 0^{p(u, v)} a
\end{array}\right)=0 \quad \forall a \in A_{U_{\beta}}
$$

where $p(u, v)$ is defined using Proposition 19. In this extended context, Proposition 26. Proposition 27. Corollary 28, Lemma 29 and Remark 30 are easy to adapt. Note that the pairs $(u, v)$ satisfying this condition depend on the choice of $q$ and $r$. The sets $\mathcal{A}_{n}^{\beta}$ are defined as before. The pair $(u, u)$ satisfies $(\star)_{r}$ if and only if $r=1$; see Lemma 25. Thus, the segment of slope 1 with endpoints $(0,0)$ and $(1,1)$ belongs to $\mathcal{A}_{0}^{\beta}$ if and only if $r=1$. An alternative proof of Proposition 36 follows the same lines as in [10].

\section{Appendix}

Example 48. We have represented the set $\mathcal{U}_{9}^{\varphi}$ in Figure 14.

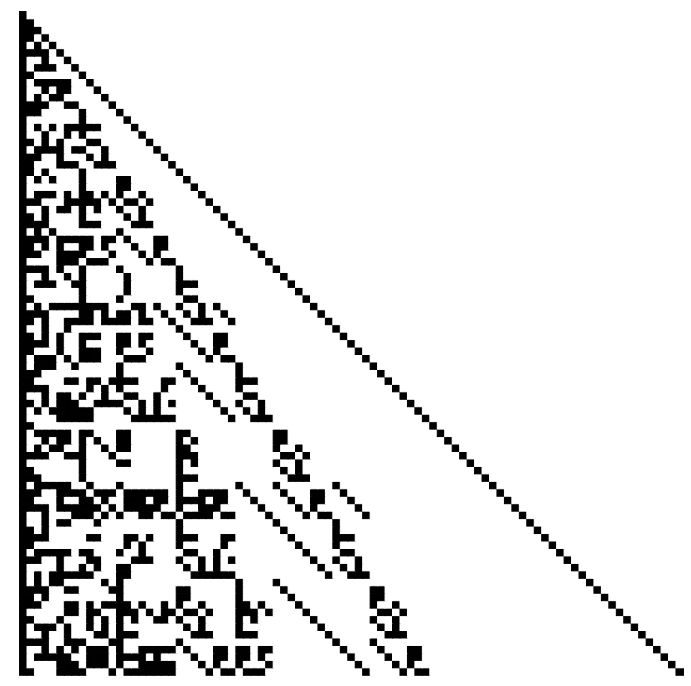

Figure 14: The set $\mathcal{U}_{9}^{\varphi}$.

Example 49. Let us consider the case when $\beta=\varphi$ is the golden ratio. We have represented in Figure 15 the set $\mathcal{U}_{9,2}^{\varphi}$ when considering binomial coefficients congruent to 2 modulo 3 and an approximation of the limit set $\mathcal{L}^{\varphi}$ proceeding as in Example 38 .

In this last example, we give an approximation of the limit object $\mathcal{L}^{\beta}$ for several different values of $\beta$. A real number $\beta>1$ is a Pisot number if it is an algebraic integer whose conjugates have modulus less than 1 . 

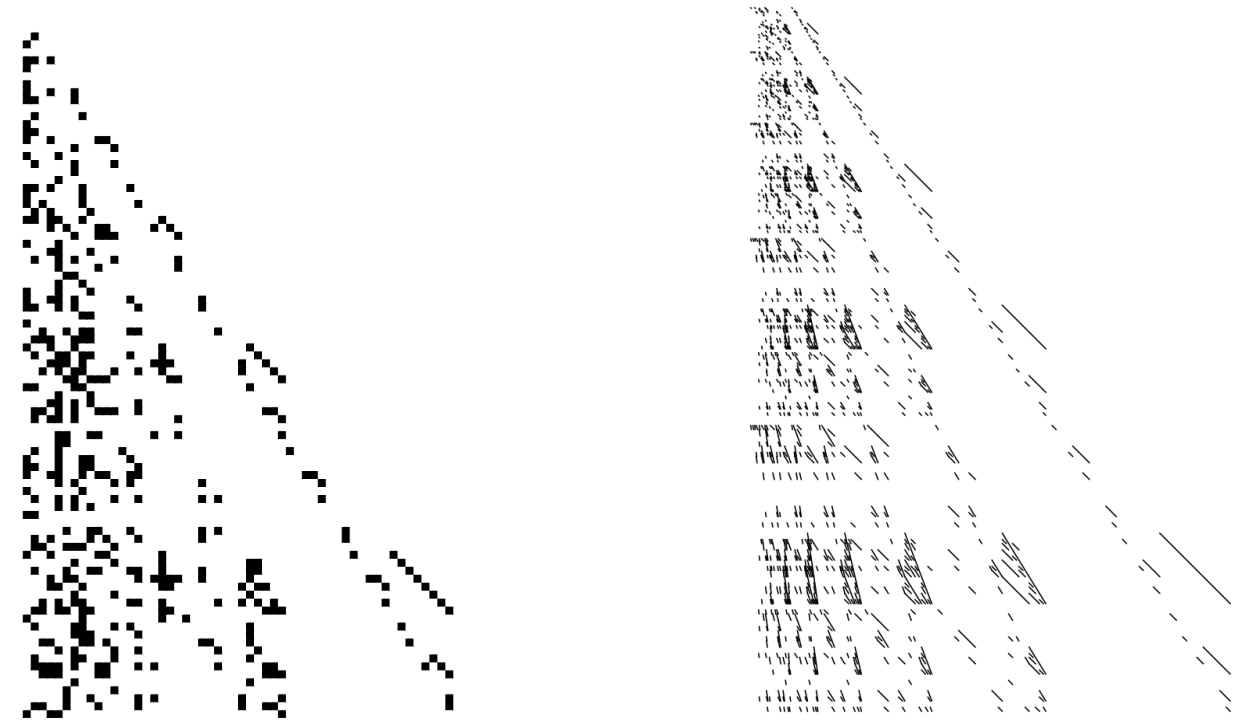

Figure 15: The set $\mathcal{U}_{9,2}^{\varphi}$ (on the left) and an approximation of the corresponding limit set $\mathcal{L}^{\varphi}$ (on the right).

Example 50. Let us define several Parry numbers. Let $\beta_{1} \approx 2.47098$ be the dominant root of the polynomial $P(X)=X^{4}-2 X^{3}-X^{2}-1$, which is a Parry and Pisot number; see Example 22. Let $\beta_{2} \approx 2.47098$ be the dominant root of the polynomial $P(X)=X^{4}-X^{3}-1$, which is a Parry and Pisot number; see Example 43 Let $\beta_{3} \approx 2.80399$ be the dominant root of the polynomial $P(X)=X^{4}-2 X^{3}-2 X^{2}-2$. We can show that $\beta_{3}$ is a Parry number, but not a Pisot number. Let $\beta_{4} \approx 1.32472$ be the dominant root of the polynomial $P(X)=X^{5}-X^{4}-1$. We can show that $\beta_{4}$ is a Parry number and also the smallest Pisot number. In Figure 16, we depict an approximation of $\mathcal{L}^{\beta}$ for $\beta$ in $\left\{\varphi^{2}, \beta_{1}, \ldots, \beta_{4}\right\}$.

\section{Acknowledgments}

This work was supported by a FRIA grant [grant number 1.E030.16].

The author wants to thank her advisor, Michel Rigo, and her colleague, Julien Leroy, for interesting scientific conversations, very useful comments on and improvements to a first draft of this paper.

\section{References}

[1] H. Belbachir, L. Németh, and L. Szalay, Hyperbolic Pascal triangles, Appl. Math. Comput. 273 (2016), 453-464.

[2] H. Belbachir and L. Szalay, On the arithmetic triangles, Šiauliai Math. Semin. 9 (2014), no. 17, 15-26.

[3] V. Berthé, M. Rigo (Eds.), Combinatorics, automata and number theory, Encycl. of Math. and its Appl. 135, Cambridge University Press, 2010.

[4] A. Bertrand-Mathis, Comment écrire les nombres entiers dans une base qui n'est pas entière, Acta Math. Hungar 54 (1989), 237-241.

[5] É. Charlier, N. Rampersad, M. Rigo, L. Waxweiler, The minimal automaton recognizing $m \mathbb{N}$ in a linear numeration system, Integers 11B (2011), Paper No. A4, 24 pp. 
[6] K. Falconer, The Geometry of Fractal Sets, Cambridge University Press, New York, 1985.

[7] N. Fine, Binomial coefficients modulo a prime, Amer. Math. Monthly 54 (1947), 589-592.

[8] F. von Haeseler, H.-O. Peitgen, and G. Skordev, Pascal's triangle, dynamical systems and attractors, Ergod. Th. E Dynam. Sys. 12 (1992), 479-486.

[9] É. Janvresse, T. de la Rue, and Y. Velenik, Self-similar corrections to the ergodic theorem for the Pascal-adic transformation, Stoch. Dyn. 5 (2005), no. 1, 1-25.

[10] J. Leroy, M. Rigo, and M. Stipulanti, Generalized Pascal triangle for binomial coefficients of words, Adv. in Appl. Math. 80 (2016), 24-47.

[11] J. Leroy, M. Rigo, and M. Stipulanti, Counting the number of non-zero coefficients in rows of generalized Pascal triangles, Discrete Math. 340 (2017), 862-881.

[12] J. Leroy, M. Rigo, and M. Stipulanti, Behavior of digital sequences through exotic numeration systems, Electron. J. Combin. 24 (2017), no. 1, Paper 1.44, 36 pp.

[13] J. Leroy, M. Rigo, and M. Stipulanti, Counting Subword Occurrences in Base- $b$ Expansions, to appear in Integers.

[14] M. Lothaire, Combinatorics on Words, Cambridge Mathematical Library, Cambridge University Press, 1997.

[15] M. Lothaire, Algebraic Combinatorics on Words, Encyclopedia of Mathematics and Its Applications, Cambridge University Press, vol. 90, 2002.

[16] É. Lucas, Théorie des fonctions numériques simplement périodiques, Amer. J. Math. 1 (1878) 197-240.

[17] W. Parry, On the $\beta$-expansions of real numbers, Acta Math. Acad. Sci. Hungar. 11 (1960), 401-416.

[18] M. Rigo, Formal languages, automata and numeration systems. 1. Introduction to combinatorics on words, ISTE, London; John Wiley \& Sons, Inc., Hoboken, NJ, 2014.

[19] M. Rigo, Formal languages, automata and numeration systems. 2. Applications to recognizability and decidability, ISTE, London; John Wiley \& Sons, Inc., Hoboken, NJ, 2014.

[20] N. J. A. Sloane, The On-Line Encyclopedia of Integer Sequences. Published electronically at http: //oeis.org, 2017. 


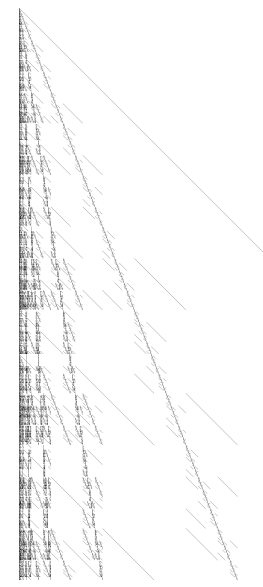

(a) An approximation of $\mathcal{L}^{\varphi^{2}}$.

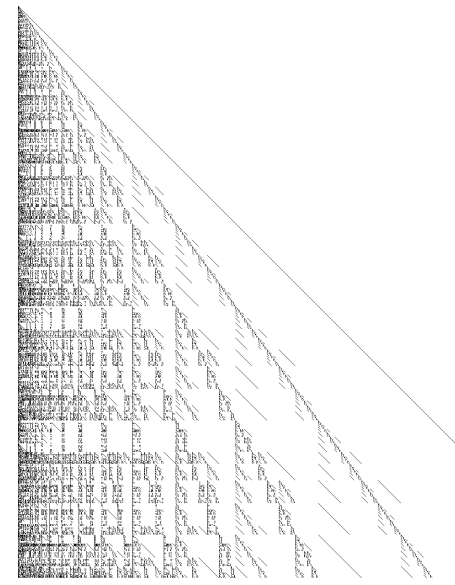

(c) An approximation of $\mathcal{L}^{\beta_{2}}$.

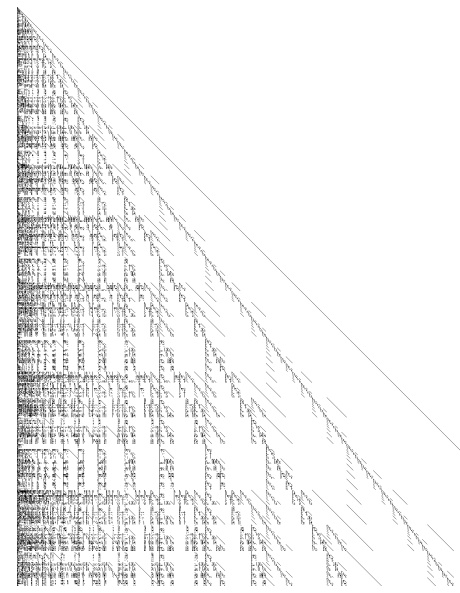

(e) An approximation of $\mathcal{L}^{\beta_{4}}$. (b) An approximation of $\mathcal{L}^{\beta_{1}}$.

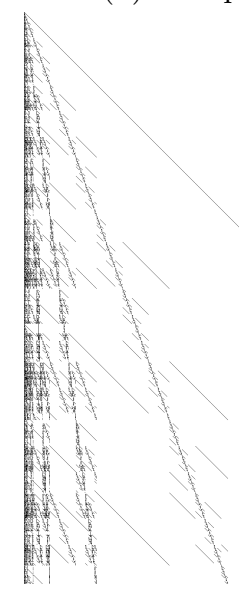

(d) An approximation of $\mathcal{L}^{\beta_{3}}$.

Figure 16: An approximation of the limit object $\mathcal{L}^{\beta}$ for different values of $\beta$. 